\title{
LÉVY AREA OF WIENER PROCESSES IN BANACH SPACES
}

\author{
By M. LEDOUX, T. LYONS AND Z. QIAN \\ University of Toulouse, CNRS and University of Oxford
}

The goal of this paper is to construct canonical Lévy area processes for Banach space valued Brownian motions via dyadic approximations. The significance of the existence of canonical Lévy area processes is that a (stochastic) integration theory can be established for such Brownian motions (in Banach spaces). Existence of flows for stochastic differential equations with infinite dimensional noise then follows via the results of Lyons and Lyons and Qian [see, e.g., System Control and Rough Paths (2000). Oxford Univ. Press]. This investigation involves a careful analysis on the choice of tensor norms, motivated by the applications to infinite dimensional stochastic differential equations.

1. Introduction. The archetypical controlled system is described by a differential equation:

$$
d y_{t}=f\left(y_{t}\right) d x_{t}, \quad y_{0}=a .
$$

We are interested in the case where $y=\left(y_{t}\right)$, which represents the state of the system, takes its values in a Banach space $E$; the controlling process $x=\left(x_{t}\right)$ takes its values in a second Banach space $B$. For any $b \in B, f(\cdot) b$ defines a vector field on $E$ which varies linearly with $b \in B$.

If the vector field $f$ is Lipschitz:

$$
\begin{aligned}
|f(e) b|_{E} & \leq C|b|_{B}, \\
\left|f(e) b-f\left(e^{\prime}\right) b\right|_{E} & \leq C\left|e-e^{\prime}\right|_{E}|b|_{B}
\end{aligned}
$$

and $x$ has bounded variation (on $[0, T]$ ):

$$
\sup _{0 \leq t_{1} \leq \cdots \leq t_{n} \leq T} \sum_{i=1}^{n}\left|x_{t_{i}}-x_{t_{i-1}}\right|_{B}<\infty,
$$

then the equation (1.1) has a unique solution. In generality, the map taking $x$ to $y$ is often known as the Itô map.

In the finite dimensional setting, Itô extended the definition of (1.1) to the case where $x=\left(x_{t}\right)$ is a vector valued Brownian motion, and Itô's technique combined with martingale techniques permitted the extension to the case where $x=\left(x_{t}\right)$ is a semimartingale.

Received April 2000; revised May 2001.

Supported by TMR Grant GRBFMRXCT960075.

AMS 2000 subject classifications. Primary 60H10; secondary 60H15, 60J60, 60G15.

Key words and phrases. Brownian motion, differential equation, Gaussian comparison theorem, Gaussian measure, rough path. 
More recently, the standard Ito theory has been extended to the case where $E$ and $B$ are $M$-type spaces (essentially Banach spaces that are similar to $L^{p}$-spaces, $p \geq 2$ ) by Brzez'niak and colleagues (see [3]). The key point is that for those Banach spaces the norm $\left|M_{t}\right|^{p}$ of a Banach space valued martingale $\left(M_{t}\right)$ is still controlled by $\mathbf{E}\left(\langle M\rangle_{t}^{p / 2}\right)$, where $\langle M\rangle_{t}$ is the scalar quantity

$$
\lim _{n \rightarrow \infty} \sum_{k=1}^{\left[2^{n} t\right]}\left|M_{k / 2^{n}}-M_{(k-1) / 2^{n}}\right|^{2} .
$$

The usual finite dimensional arguments can be made to carry over to this case.

In a slightly different direction, Walsh [29] and DaPrato and Zabczyk [9], etc., have considered stochastic perturbations to dissipative evolution equations (e.g., parabolic stochastic partial differential equations). In this setting $B$ is densely embedded in $E$ and $f$ is a dissipative vector field such that the flow defined by

$$
d y_{t}=f\left(y_{t}\right) d t, \quad \pi_{s t}\left(y_{s}\right)=y_{t}
$$

extends to a differentiable map from $E$ to $B$ for each $s<t$. If $x$ is a Brownian motion on $E$, one may consider perturbing the deterministic flow to the controlled equation

$$
d \tilde{y}_{t}=f\left(\tilde{y}_{t}\right) d t+d x_{t} .
$$

Interpreting this equation in mild form

$$
\tilde{y}_{t}=\pi_{0 t}\left(\tilde{y}_{0}\right)+\int d \pi_{s t}\left(\tilde{y}_{s}\right) d x_{t},
$$

and where the integral is interpreted in the Itô sense. In the case where $f$ is linear in $y$ and we denote the generated semigroup $e^{t f}$ as $P_{t}$, the mild equation becomes

$$
\tilde{y}_{t}=P_{t}\left(\tilde{y}_{0}\right)+\int_{0}^{t} P_{t-s}\left(d x_{s}\right)
$$

and one quickly sees that a sufficient condition in the case that $B$ is an $M$-type space for the existence of a solution of the equation is that

$$
\int_{0}^{t}\left\|P_{s}\right\|_{B \rightarrow E}^{2} d s<\infty .
$$

However, many extremely fundamental questions of a simple nature are still open. We mention one. The existence of solutions to bounded linear stochastic equations. Let $f(\cdot) b: E \rightarrow E$ be a bounded linear map for each $b$ satisfying:

$$
|f(e) b|_{E} \leq K|b|_{B}|e|_{E},
$$

and suppose $x=\left(x_{t}\right)$ is a Brownian motion with values in $B$. Can one, with probability one, solve equation (1.1)? 
A special case is where $x$ is a Brownian motion on a Banach space $B$, and $E=\mathbf{R} \oplus B \oplus(B \otimes B)$ with the projective tensor product on $B \otimes B$. Consider the (noncommutative) exponential style equation

$$
d y_{t}=y_{t} \times d x_{t}, \quad y_{0}=a,
$$

where $e \times b=e \otimes b \in B \oplus(B \otimes B)$ if $e \in \mathbf{R} \oplus B$ and $e \times b=0$ if $e \in B \otimes B$. It is an important case because identifying a meaningful solution to (1.2) of homogenous $p$-variation in effect associates a "rough path" to $x=\left(x_{t}\right)$. Applying the theory developed in [23-25], one obtains solutions to all sufficiently smooth differential equations (1.1) driven by $x$. As linear equations are sufficiently smooth, thus it is necessary to settle equation (1.1) for linear equations before we are able to solve (1.1) for all smooth $f$.

The approach of $M$-type spaces is of frustratingly little help because the projective tensor product $B \otimes B$ is in general not a nice space even if $B$ is.

In this paper, we construct a canonically associated geometric rough path for Banach space valued Wiener process. Therefore, by the results in [23] (see also [25]), we may extend the classical Stratonovich integration theory to Banach space valued Wiener process. A theory of stochastic differential equations for such kind of infinite white noise may therefore be established accordingly and goes beyond the known ones that are restricted to families of smooth Banach spaces [3, 9]. In so doing we are able to solve a wide range of equations which were previously out of range, and recover many known examples.

By the nature of the rough path analysis, one obvious advantage of our approach over the existed results is that we may solve all stochastic differential equations on Banach spaces run by a Wiener process with a common chosen null set which depends only on the sample paths of the Wiener process. The conditions we found for Banach space valued Wiener processes such that the results in the present work can be applied seem very natural, as they only involve the intrinsic property of the marginal Gaussian measures of Wiener processes, and are rather easy to check. Indeed, at a technical level our core result in this paper is to decide some practical criteria on a Banach space $B$, and on a Gaussian measure $\mu$ supported on $B$, so that the Brownian motion $x=\left(x_{t}\right)$ with increments given by $\mathbf{P}\left((t-s)^{-1 / 2}\left(x_{t}-x_{s}\right) \in A\right)=\mu(A)$ has a canonical Lévy area process. We prove it will always lie in the injective tensor product of $B$ with itself, and give simple criteria on $\mu$ for its existence within the projective tensor product. More precisely, we deal with arbitrary Gaussian measures on a Banach space $B$, but however do impose restrictions on the choice of the tensor norm on $B \otimes B$, that in turn impose regularity on the input of the SDE. Alternatively, we restrict the Gaussian measures on $B$ that generates the Wiener processes but deal with arbitrary tensor norms.

However, we are not claiming the approach presented here covers all known results. Indeed, the general results in [23] require that the vector fields are $\operatorname{Lip}(\gamma)$ maps for some $\gamma>p$ for the existence and the uniqueness (and therefore the 
existence of the flows), and thus applying to the case treated here, we require the vector fields are $C^{2+\epsilon}$ for some $\epsilon>0$. While, in the circulated papers [4, 5], under the setting of $M$-type Banach spaces, the authors are able to solve SDE with Lipschitz vector fields. Davie has recently informed the second author that he is able to prove the existence of solutions for differential equations with $\operatorname{Lip}(\gamma-1)$ vector fields, driven by general geometric rough paths of $p$-variations, provide $\gamma>p$, while under only these conditions, the uniqueness is no longer true. When the controlling path is Brownian motion in the Euclidean space, he is able to carry out this rough path approach under least smoothness conditions on the vector fields. We thus could hope that our results might be improved.

The paper is organized as follows. In Section 2 we remind the reader of the main results we need on rough paths. In Section 3, we introduce Brownian motions and Wiener processes in Banach spaces and collect some general facts from the theory of Gaussian processes. Section 4 contains technical results to control variation distances. In Section 5 we present a general procedure to construct a canonical Lévy area process and geometric rough path associated to a Wiener process and introduce in particular exact tensor norms for which the construction applies. Section 6 develops applications of the previous section and examples of tensor norms and underlying Gaussian measures for which the results apply. In particular, we show that the Lévy area always lies in the injective tensor product.

2. Rough paths and Lévy area. The Itô map defined by taking the classical solution $y$ to

$$
d y_{t}=f\left(y_{t}\right) d x_{t}, \quad y_{0}=a,
$$

is well defined and even continuous in total variation norm providing the vector field is Lipschitz in a Banach space $B$. However it is only in the most trivial and well understood situations that it is continuous with respect to the topology of uniform convergence.

This provided an obstacle to extending the Itô map to paths with less regularity such as Brownian paths. However, the main result in [23] shows that the Itô functional is uniformly continuous in " $p$-variation" metric for every $p \geq 1$, providing $f$ is $\operatorname{Lip}(\gamma)$ with $\gamma>p$ and for $p \geq 2$ that the $p$-variation metric is appropriately defined. Recall that, in general we say a continuous map $f$ from a Banach space $V$ to a Banach space $W$ is $\operatorname{Lip}(\gamma)$ with respect to Banach tensor products $V^{\otimes i}$ and $W^{\otimes i}$ (for $i=1, \ldots,[\gamma]$ ), if there are continuous maps $f^{i}: V \rightarrow L\left(V^{\otimes i}, W^{\otimes i}\right)$ [where $L\left(V^{\otimes i}, W^{\otimes i}\right)$ denotes the Banach space of all linear maps from $V^{\otimes i}$ into $W^{\otimes i}$ ] and maps $R_{i}: V \times V \rightarrow L\left(V^{\otimes i}, W\right)$ such that

$$
\begin{gathered}
f^{j}\left(x_{t}\right)=\sum_{i=0}^{[\gamma]-j} f^{i+j}\left(x_{s}\right)\left(x_{s, t}^{i}\right)+R_{j}\left(x_{s}, x_{t}\right), \\
\left|R_{j}(v, w)\right| \leq M|v-w|^{\gamma-[\gamma]} ; \quad\left|f^{j}(v)\right| \leq M(1+|v|)
\end{gathered}
$$


for all $j \leq[\gamma]$ (for some constant $M$ ), and all continuous path $\left(x_{t}\right)$ with finite total variation in $V$. Of course it is required that $f^{1}=f$, and the reader can see the candidate for $f^{j+1}$ is the $j$ th Fréchet derivative of $f$ (when such a derivative exists). The above definition not only involves the Banach spaces $V$ and $W$, but also their tensor products up to degree $[\gamma]$.

If $x$ is a continuous path in a vector space, one may define its piecewise linear dyadic approximations: if $n \in \mathbf{N}, k=2^{n-1}+1, \ldots, 2^{n}$ and $\theta \in[0,1]$, we define

$$
x(n)_{\theta k / 2^{n}+(1-\theta)(k-1) / 2^{n}}=\theta x_{k / 2^{n}}+(1-\theta) x_{(k-1) / 2^{n}} .
$$

Then $x(n)$ is of bounded variation on finite intervals and so is in the domain of the Itô functional. Let $y(n)$ be defined by

$$
d y(n)_{t}=f\left(y(n)_{t}\right) d x(n)_{t}, \quad y(n)_{0}=a .
$$

Providing that $(x(n))$ is a Cauchy sequence in $p$-variation metric, the uniform continuity implies that $(x(n), y(n))$ is also Cauchy, and hence our equation has a natural solution as a rough path $(x, y)$ of finite $p$-variation.

This strategy has been carried out for almost all Brownian paths in Euclidean spaces by Sippilainen [26], where convergence occurs for any $p>2$. The papers $[2,8,16]$ establish the same sort of results for a wide range of diffusion processes and also for fractional Brownian motions. The main restriction seems to be the requirement that the sample paths have $p$-variation for $p$ less than 4 . One attraction is the common null set for all smooth enough differential equations.

We extend the approach to the "simple" case of a Gaussian Brownian motion (and therefore with finite $p$-variation for any $p>2$, so we restrict to $2<p<3$ ) on a Banach space. It turns out that this not in any way a trivial extension of the finite dimensional case and depends sensitively on the existence of quadratic functionals of the path in particular tensor products of the Banach space $B$ with itself.

The reason for this will be apparent when the reader understands the definition of $p$-variation we use here and also thinks through the meaning of $\operatorname{Lip}(\gamma)$ if $\gamma>1$ (which is required for irregular paths like Brownian ones).

Suppose $x=\left(x_{t}\right)$ is a path of bounded variation in $B$. Then we may compute for $s<t$ its iterated integrals:

$$
\begin{aligned}
x_{s, t}^{1} & =\int_{s<u<t} d x_{u}, \\
x_{s, t}^{2} & =\int_{s<u_{1}<u_{2}<t} d x_{u_{1}} \otimes d x_{u_{2}}, \\
& \vdots \\
x_{s, t}^{i} & =\int_{s<u_{1}<\cdots<u_{i}<t} d x_{u_{1}} \otimes \cdots \otimes d x_{u_{i}} .
\end{aligned}
$$

Of course $x_{s, t}^{1}=x_{t}-x_{s} \in B$, but $x_{s, t}^{i}$ lives in some sort of tensor product for $i>1$. 
Let $|\cdot|_{B^{\otimes i}}$ be any norm on the algebraic tensor product $B^{\otimes i}$ with the property that if $u \in B^{\otimes i}$ and $v \in B^{\otimes j}$ then

$$
|u \otimes v|_{B^{\otimes(i+j)}} \leq|u|_{B^{\otimes i}}|v|_{B^{\otimes j}} .
$$

Then

$$
\left|x_{s, t}^{i}\right|_{B^{\otimes i}} \leq \frac{A C^{i}}{i !}
$$

where $A$ and $C$ are numbers related to the total variation of $x$ over the intervals $[s, t]$ alone.

The $p$-variation distance between two paths $x$ and $y$ (on some interval $[0, T]$ ) is defined by

$$
d_{p}(x, y)=\sum_{i=1}^{[p]}\left(\sup _{D} \sum_{l}\left|x_{t_{l-1}, t_{l}}^{i}-y_{t_{l-1}, t_{l}}^{i}\right|_{B}^{p / i}\right)^{i / p}+\sup _{u \in[0, T]}\left|x_{u}-y_{u}\right|
$$

where $D$ is running over all finite partitions $\left(t_{l}\right)$ of $[0, T]$. A geometric rough path of finite $p$-variation is, by definition, a point in the completion of the bounded variation paths under this metric $d_{p}$, and has a unique representation as a sequence

$$
\mathbf{x}=\left(\left(1, x_{s, t}^{1}, x_{s, t}^{2}, \ldots, x_{s, t}^{[p]}\right) ; 0 \leq s \leq t \leq T\right)
$$

and can be identified with a path in the truncated tensor algebra

$$
T^{([p])}(B) \equiv \mathbf{R} \oplus B \oplus B^{\otimes 2} \oplus \cdots \oplus B^{\otimes[p]}
$$

having certain special properties. While $x_{s, t}^{1}=x_{t}-x_{s}$, the second iterated integrals $x_{s, t}^{2}$ define the "Lévy area process" of the curve $\left(x_{t}\right)$.

The continuity result in [23] allows one to construct solutions to equations such as (1.1) for any rough paths, providing $f$ is smooth enough. Associated results enable one to give meaning to the equation.

We are now in a position to understand the subtlety arising for even the classical Brownian motion in an infinite dimensional space. If $x$ is a Brownian path in the Banach space $B$, then we would like to consider the sequence $x(n)$ of piecewise smooth approximations to $x$, and prove they are Cauchy in the metric $d_{p}$ for some $p$. This will be impossible unless $p>2$. Once $p$ is greater then 2 we have to consider the second iterated integral (Lévy area) and estimate its norm. But which norm? The definition of the metric $d_{p}$ (for $p>2$ ) depends on choosing a tensor norm for the algebraic tensor product $B \otimes B$. Such norms have been extensively studied and there is a smallest (the injective norm producing the biggest Banach space: the injective tensor product), and a largest (the projective norm producing the smallest Banach space: the projective tensor product).

The choice of norm on the algebraic tensor product $B \otimes B$ matters (and not for a technical reason) because it is implicit in the definition of $\operatorname{Lip}(\gamma)$ property for $\gamma>p$ (see $[23,25])$. The definition of $\operatorname{Lip}(\gamma)$ property involves the boundedness 
of a linear map on $B \otimes B$ that arose from the second differentiation. The boundedness requirement is dual to the tensor norm used in the definition of the metric $d_{p}$.

Choosing a smaller norm on the space $B \otimes B$ requires that $f$ has a "more bounded" Hessian, which will restrict the class of differential equations we are able to solve. For this reason it would be ideal if one could prove that $x(n)$ converges to $x$ in $d_{p}(2<p<3)$ with the projective norm on $B \otimes B$. In this case all equations (1.1) with regular vector field $f$ (especially all linear equations) are solvable for $(x, B)$. (However we do not believe it always happens, and in consequence conjecture that our problem has a negative answer: there will be some $(x, B)$ for which not all linear equations are solvable.) In our main Theorem 3 together with the results of Section 5, we will prove that in standard examples of distribution $\mu, x(n) \rightarrow x$ in $d_{p}(2<p<3)$ with the projective norm on $B \otimes B$. That is, we prove the existence of a path $\mathbf{x}=\left(1, x^{1}, x^{2}\right)$ in $\mathbf{R} \oplus B \oplus B^{\otimes 2}$ such that $\mathbf{x}(n)=\left(1, x^{1}(n), x^{2}(n)\right) \rightarrow \mathbf{x}$ in $d_{p}$ where $x_{s, t}^{1}(n)=x(n)_{t}-x(n)_{s}, s<t$. Similarly $x_{s, t}^{1}=x_{t}-x_{s}$ while the second level path $x_{s, t}^{2}$ defines the Lévy area of the vector valued Brownian motion $x$. On the other hand we are able to show (cf. Theorem 4) that for any $(x, B)$ the second iterated integrals converge to a Lévy area process, and thus the sequence $x(n)$ converges to $x$, in the metric $d_{p}$ for any $2<p<3$, provided the norm on $B \otimes B$ is the injective one, so the iterated integrals exist in the larger space.

These results yield the following consequences to the study of the differential equation (1.1). If $f$ is Fréchet differentiable (up to degree 3), denote by $F(x(n), \cdot, a)=y(n)+a$ the solution of the system $d y(n)_{t}=f\left(y(n)_{t}\right) d x(n)_{t}$, $y(n)_{0}=a$. Denote by $y(n)_{s, t}^{i}$ the $i$ th iterated path integral of $F(x(n), \cdot, a)$ over $[s, t]$. As explained in $[23,25]$, any such $F$ is Lipschitz if $B \otimes B$ is the projective tensor product, and therefore by the universal limit theorem in [23, 24], we may state:

COROLlaRY 1. Suppose that for some distribution $\mu, x(n) \rightarrow x$ in $d_{p}(2<$ $p<3)$ with the projective norm on $B \otimes B$. Suppose $f$ is $C^{3}$ in the Fréchet sense. Then $y(n) \rightarrow y$ in $d_{p}(2<p<3)$ with the projective norm on $E \otimes E$. That is, there is a unique path $\mathbf{y}=\left(1, y^{1}, y^{2}\right)$ in $\mathbf{R} \oplus E \oplus E^{\otimes 2}$ (with $E^{\otimes 2}$ equipped with the projective topology) such that $\mathbf{y}(n)=\left(1, y^{1}(n), y^{2}(n)\right) \rightarrow \mathbf{y}$ in $d_{p}, 2<p<3$, almost surely. In particular, $F(x(n), t, a) \rightarrow y_{0, t}^{1}+a$ (denoted by $F(x, t, a)$ ) uniformly in $t$ and $F(x, t, a)$ can be regarded as the strong solution $y_{t}+a$ of equation (1.1). Moreover if $f$ is smooth, then for any $t$, the map $a \rightarrow F(x, t, a)$ forms a flow of diffeomorphisms.

Although one can not solve all (even linear) equations like (1.1) via the Lévy area process in the injective tensor product, it allows us to solve (1.1) when the target space $E$ is a finite dimensional space. Namely, when $E$ is finite dimensional, 
it is easily checked that any Fréchet differentiable function $f: E \rightarrow \mathcal{L}(B, E)$ is Lipschitz with respect to any Banach tensor product $B \otimes B$. Since we can show that $x(n) \rightarrow x$ in $d_{p}(2<p<3)$ for the injective norm on $B \otimes B$ for any underlying Gaussian distribution $\mu$ (Theorem 4), the results in [23] apply similarly in this case to yield:

COROLlary 2. If $f$ is Fréchet differentiable up to degree 3, and if $E$ is finite dimensional Banach space, then for any Brownian motion $x$ in $B$, the conclusions in the previous corollary remain true.

For some special Banach spaces such as $L^{p}(\Sigma)$-spaces for $1 \leq p<\infty$, one can also show that the geometric rough path $\mathbf{x}$ is always carried by an intermediate tensor product $L^{p}(\Sigma \times \Sigma)$ between the injective and the projective products.

Recent related developments in the context of free probability are investigated in [6].

3. Wiener processes. In this section, we briefly recall the definitions of abstract Wiener spaces and Banach space valued Wiener processes following the references $[14,17]$. We then present the entropy and comparison theorems for Gaussian processes (cf. [19, 20, 22]).

3.1. Abstract Wiener spaces and B-valued Wiener processes. An abstract Wiener space $[14,17]$ is by definition a triple $(B, H, \mu)$ which consists of a separable Banach space $B$ with norm $|\cdot|_{B}$, a separable Hilbert space $H$ which is a vector subspace of $B$ with the canonical embedding $i: H \hookrightarrow B$, and a Gaussian measure $\mu$ on the Borel $\sigma$-field $\mathcal{B}$ of $B$ with covariance $H$. For simplicity, we only consider throughout this work centered Gaussian measures. Thus $\mu$ is the unique probability measure on $(B, \mathscr{B})$ such that each continuous linear functional $\xi$ on $B$ has normal distribution $\mathcal{N}\left(0,|\xi|_{H}^{2}\right)$. For any $\xi \in B^{*} \hookrightarrow H$, define the pairing $j_{\xi}: B \rightarrow \mathbf{R}$ between $B^{*}$ and $B$ by $j_{\xi}(x)=\langle x, \xi\rangle$. Then $\mathcal{B}$ is the smallest $\sigma$-field such that all functions $j_{\xi}$ are measurable and $\mu$ is the unique probability measure on $(B, \mathscr{B})$ such that

$$
\int_{B} \exp (i\langle x, \xi\rangle) \mu(d x)=\exp \left(-\frac{1}{2}|\xi|_{H}^{2}\right)
$$

for any $\xi \in B^{*}$. The coordinate function $e(x)=x$ is a $B$-valued Gaussian random variable under the probability $\mu$.

An important property is the existence and equivalence of moments of Gaussian measures due to Fernique [11] and Landau and Shepp [18] (cf. [19, 20, 22]). In particular, for any $p, q>0$, there is a constant $C_{p, q}$ only depending on $p$ and $q$ such that

$$
\left(\int_{B}|x|_{B}^{p} \mu(d x)\right)^{1 / p} \leq C_{p, q}\left(\int_{B}|x|_{B}^{q} \mu(d x)\right)^{1 / q}<\infty .
$$


Let $G$ be a $B$-valued Gaussian random variable on some probability space $(\Omega, \mathcal{F}, \mathbf{P})$ with distribution $\mu$. In other words, $G: \Omega \rightarrow B$ is a random variable on $(\Omega, \mathcal{F}, \mathbf{P})$ such that for any $\xi \in B^{*},\langle G, \xi\rangle$ is a centered Gaussian real random variable with variance $|\xi|_{H}^{2}$. The next lemma (see, e.g., [20], Proposition 4.2.) is a standard useful fact.

LEMMA 1. Let $\left\{\xi_{i}\right\}(i=1,2, \ldots)$ be an orthonormal basis of $H$ such that $\xi_{i} \in B^{*}$, and let $G$ be a $B$-valued centered Gaussian random variable on $(\Omega, \mathcal{F}, \mathbf{P})$ with distribution $\mu$. Set $g_{i}=\left\langle G, \xi_{i}\right\rangle$. Then $\left\{g_{i}\right\}$ is a sequence of independent random variables on $(\Omega, \mathcal{F}, \mathbf{P})$ with standard normal distribution $\mathcal{N}(0,1)$ and

$$
G=\sum_{i=1}^{\infty} g_{i} \xi_{i} \quad \text { almost surely in } B \text { and in } L^{p}(\Omega ; B) .
$$

The prime example of an abstract Wiener space is the standard Wiener space where $B$ is the space of continuous functions on $[0,1]$ say, $H$ is the classical Cameron-Martin Hilbert space and $\mu$ is the Wiener measure. This model thus gives rise to the Wiener process or Brownian motion $X=\left(X_{t}\right)_{t \geq 0}$ with values in $\mathbf{R}$ or $\mathbf{R}^{d}$. The construction of the classical Wiener process may be extended to paths with values in a (infinite dimensional separable) Banach space. More precisely, standard references such as, for example, $[15,17]$ [or simply the classical approach together with the basic existence (3.1) of moments] show that, given an abstract Wiener space $(B, H, \mu)$, there exists a stochastic process $X=\left(X_{t}\right)_{t \geq 0}$ on some probability space $(\Omega, \mathcal{F}, \mathbf{P})$ with values in $B$ such that $X_{0}=0$ almost surely, $X$ has independent increments and, for any $s<t$, and any $\xi \in B^{*}$, the increment $\left\langle X_{t}-X_{s}, \xi\right\rangle$ has normal distribution $\mathcal{N}\left(0,(t-s)|\xi|_{H}^{2}\right)$. In other words, $X_{1}$ has distribution $\mu$ and $X_{t}-X_{s}$ has the same distribution as $(t-s)^{1 / 2} X_{1}$. Moreover, almost all sample paths $t \rightarrow X_{t}$ are continuous from $\mathbf{R}_{+}$to $B . X=\left(X_{t}\right)_{t \geq 0}$ is called Wiener process based on (the abstract Wiener space) $(B, H, \mu)$, or $B$-valued Wiener process generated by $\mu$.

Since $X_{t}-X_{s}, s<t$, has the same distribution as $(t-s)^{1 / 2} X_{1}$, and since $X_{1}$ has distribution $\mu$, note that by (3.1),

$$
\mathbf{E}\left(\left|X_{t}-X_{s}\right|_{B}^{p}\right)=C_{p}|t-s|^{p / 2}
$$

where $C_{p}=\int_{B}|x|^{p} \mu(d x)<\infty$ for every $0<p<\infty$.

3.2. Gaussian processes. A family of real valued random variables $G=$ $\left(G_{t}\right)_{t \in T}$ on a probability space $(\Omega, \mathcal{F}, \mathbf{P})$ (where $T$ can be any index set) is called a centered (real valued) Gaussian process if for any $t_{1}, \ldots, t_{k}$ in $T,\left(G_{t_{1}}, \ldots, G_{t_{k}}\right)$ possesses centered Gaussian distribution on $\mathbf{R}^{k}$. 
The modern investigation $[19,22]$ of boundedness and continuity of general Gaussian processes $G=\left(G_{t}\right)_{t \in T}$ is based on the analysis of the size of the parameter set $T$ for the $L^{2}$-metric (or pseudo-metric) induced by $G$,

$$
d(s, t)=d_{G}(s, t)=\left(\mathbf{E}\left|G_{t}-G_{s}\right|^{2}\right)^{1 / 2}, \quad s, t \in T .
$$

For any $\varepsilon>0$, denote by $N(T, d ; \varepsilon)$ the minimal number of (open) balls with radius $\varepsilon$ for the metric $d$ that are necessary to cover $T$. It was shown by Dudley [10] that for some numerical constant $C>0$,

$$
\mathbf{E}\left(\sup _{t \in T} G_{t}\right) \leq C \int_{0}^{\infty} \sqrt{\log N(T, d, \varepsilon)} d \varepsilon .
$$

Moreover, if the entropy integral is finite, the Gaussian process $G$ has a version with almost all sample paths continuous with respect to $d$.

We also need to quote the important comparison theorems going back to the Slepian lemma. We use it in the form put forward by Sudakov and Fernique (cf. $[19,22])$.

THEOREM 1. Let $G=\left(G_{t}\right)_{t \in T}$ and $G^{\prime}=\left(G_{t}^{\prime}\right)_{t \in T}$ be centered Gaussian processes with $L^{2}$-metrics $d_{G}$ and $d_{G}^{\prime}$ such that, for all $s, t$ in $T$,

$$
d_{G}(s, t) \leq d_{G^{\prime}}^{\prime}(s, t) \text {. }
$$

Then

$$
\mathbf{E}\left(\sup _{s, t \in T}\left(G_{s}-G_{t}\right)\right) \leq \mathbf{E}\left(\sup _{s, t \in T}\left(G_{s}^{\prime}-G_{t}^{\prime}\right)\right)
$$

This result will be crucial in Section 5 when studying Lévy area under the injective norm.

4. Control on the $\boldsymbol{p}$-variation distance. We first recall in this section some basic notions about rough paths. Let $B$ be a real separable Banach space with norm $|\cdot|_{B}$. Denote by $T^{(2)}(B)$ the truncated tensor algebra $\mathbf{R} \oplus B \oplus(B \otimes B)$ endowed with the usual tensor product. Let $|\cdot|_{B \otimes B}$ be a tensor norm on the linear tensor product $B \otimes B$. Each element $v$ in $B \otimes B$ may be represented as $v=$ $\sum_{i=1}^{n} x_{i} \otimes y_{i}$ for $x_{i}, y_{i} \in B$ that equivalently defines a bilinear form on $B^{*} \times B^{*}$. $\left(B \otimes B,|\cdot|_{B \otimes B}\right)$ is the completion of the linear tensor product $B \otimes B$ with respect to $|\cdot|_{B \otimes B}$. At this point, we only need to know that $|x \otimes y|_{B \otimes B} \leq|x|_{B}|y|_{B}$ for $x, y \in B$. We further discuss tensor norms in Section 5 .

A multiplicative functional $\mathbf{x}$ in $T^{(2)}(B)$ is a mapping from the simplex $\Delta=$ $\{(s, t) ; 0 \leq s \leq t \leq 1\}$ into $T^{(2)}(B)$ if it can be written as $\mathbf{x}_{s, t}=\left(1, x_{s, t}^{1}, x_{s, t}^{2}\right)$ and satisfies

$$
\mathbf{x}_{s, u}=\mathbf{x}_{s, t} \otimes \mathbf{x}_{t, u} \quad \text { for any } s \leq t \leq u .
$$


Usually we only consider, for simplicity, variations on the unit interval $[0,1]$.

If we are given a smooth path $x$ in $B$, then we can define a canonical multiplicative functional $\mathbf{x}$ in $T^{(2)}(B)$ associated to $x$. Namely $x_{s, t}^{1}$ is simply the increment $x_{t}-x_{s}$ and $x_{s, t}^{2}$ is the second iterated integral

$$
x_{s, t}^{2}=\int_{s<t_{1}<t_{2}<t} d x_{t_{1}} \otimes d x_{t_{2}} .
$$

A multiplicative functional $\mathbf{x}$ in $T^{(2)}(B)$ is said to have finite $p$-variation for some $p<3$ if

$$
\sup _{D} \sum_{l}\left|x_{t_{l-1}, t_{l}}^{i}\right|_{B}^{p / i}<\infty, \quad i=1,2,
$$

where the supremum runs over all finite partitions $D=\left(t_{l}\right)$ of $[0,1]$.

Let, for any $n \in \mathbf{N}$ and $k=0,1, \ldots, 2^{n}, t_{k}^{n}=k / 2^{n}$. For each $n,\left\{t_{0}^{n}<t_{1}^{n}<\cdots\right.$ $\left.<t_{2^{n}}^{n}\right\}$ is the dyadic partition of the interval $[0,1]$ with step length $1 / 2^{n}$.

The next lemma has been put forward in [16] and is crucial in the control of the $p$-variations. We give a complete proof as we will need some of the arguments below. Once for all, we mention that we use $C$ (sometimes with a lower subscript) to denote an unimportant nonnegative constant, which may vary from line to line.

LEMMA 2. Let $\mathbf{x}$ be a multiplicative functional in $T^{(2)}(B)$. Then, for $i=1,2$, $p$ satisfying $p / i>1$, and any $\gamma>p / i-1$, there is a constant $C_{i}$ depending only on $p, \gamma, i$, such that

$$
\sup _{D} \sum_{l}\left|x_{t_{l-1}, t_{l}}^{i}\right|_{B^{\otimes i}}^{p / i} \leq C_{i} \sum_{n=1}^{\infty} n^{\gamma} \sum_{k=1}^{2^{n}} \sum_{j=1}^{i}\left|x_{t_{k-1}^{n}, t_{k}^{n}}^{j}\right|_{B^{\otimes i}}^{p / j} .
$$

PROOF. Note first that by Hölder's inequality, for any nonnegative numbers $a_{n}, n \geq 1$, and any $\gamma>q-1>0$,

$$
\left(\sum_{n=1}^{\infty} a_{n}\right)^{q} \leq\left(\sum_{n=1}^{\infty} \frac{1}{n^{\gamma / q-1}}\right)^{q-1} \sum_{n=1}^{\infty} n^{\gamma} a_{n}^{q}=C(q, \gamma) \sum_{n=1}^{\infty} n^{\gamma} a_{n}^{q}
$$

The main idea in the proof is that we can cover any subinterval $[s, t]$ of $[0,1]$ by dyadic intervals (may be infinitely many) with maximum lengths, which we shall make precise later on.

Let first $n_{0}$ be the smallest $n$ such that $[s, t]$ contains a dyadic interval $\left[t_{k}^{n}, t_{k+1}^{n}\right]$. Let $\left[t_{k_{n}}^{n_{0}}, t_{k_{n_{0}}+1}^{n_{0}}\right], 0 \leq k_{n_{0}} \leq 2^{n_{0}}-1$, be that interval. If $\left[t_{k_{n_{0}}}^{n_{0}}, t_{k_{n_{0}+1}}^{n_{0}}\right]=[s, t]$ the construction stops. If it is not the case, and if $t_{k_{n}+1}^{n_{0}}<t$, then we can carry on the construction. We may choose $n_{1}>n_{0}$ and $0 \leq k_{n_{1}} \leq 2^{n_{1}}-1$ such that $t_{k_{n_{0}}+1}^{n_{n_{0}}}=t_{k_{n_{1}}}^{n_{1}}$ and $\left[t_{k_{n_{1}}}^{n_{1}}, t_{k_{n_{1}}+1}^{n_{1}}\right]$ has maximum length among all dyadic intervals $\left[t_{k}^{n}, t_{k+1}^{n}\right] \subset$ 
$\left[t_{k_{n}+1}^{n_{0}}, t\right]$. Repeating this procedure we obtain an increasing subsequence $\left\{n_{i}\right\}$ such that

$$
t_{k_{n_{0}}}^{n_{0}}<t_{k_{n_{0}}+1}^{n_{0}}=t_{k_{n_{1}}}^{n_{1}}<t_{k_{n_{1}}+1}^{n_{1}}=t_{k_{n_{2}}}^{n_{2}}<\cdots<t_{k_{n_{i}}}^{n_{i}} \leq t
$$

with $t_{k_{n_{i}}}^{n_{i}}=t$ if the procedure ends after a finite number of steps or $t_{k_{n_{i}}}^{n_{i}} \rightarrow t$ otherwise. The same argument applies to the left-end points, and therefore we may find another increasing subsequence $\left\{n_{i}^{\prime}\right\}$ such that

$$
t_{k_{n_{0}}}^{n_{0}}>t_{k_{n_{0}}-1}^{n_{0}}=t_{k_{n_{1}^{\prime}}}^{n_{1}^{\prime}}>t_{k_{n_{1}^{\prime}}-1}^{n_{1}^{\prime}}=t_{k_{n_{2}^{\prime}}^{\prime}}^{n_{2}^{\prime}}>\cdots>t_{k_{n_{i}^{\prime}}^{\prime}}^{n_{i}^{\prime}} \geq s
$$

with $t_{k_{n_{i}^{\prime}}}^{n_{\prime}^{\prime}}=s$ or $\rightarrow s$. For simplicity let us denote by $s_{i}=t_{k_{n_{i}}}^{n_{i}}$ and $s_{-i}=t_{k_{n_{i}^{\prime}}^{\prime}}^{n_{\prime}^{\prime}}$ if $i=1,2, \ldots$ and $s_{0}=t_{k_{n_{0}}}^{n_{0}}$. Note $i \leq n_{i}, n_{i}^{\prime}$ for every $i$. Then by the above construction we have that

$$
[s, t]=\bigcup_{i \in \mathbf{Z}}\left[s_{i}, s_{i+1}\right],
$$

where the intervals $\left[s_{i}, s_{i+1}\right]$ are dyadic and disjoint (besides possibly common endpoints).

We are then in a position to prove the lemma. Start with the first level path $x^{1}$. Since $\mathbf{x}$ is multiplicative (4.1), it is natural to define

$$
x_{s, t}^{1}=\sum_{i=-\infty}^{\infty} x_{s_{i}, s_{i+1}}^{1},
$$

and we check in the following convergence of this series. By the triangle inequality and (4.2),

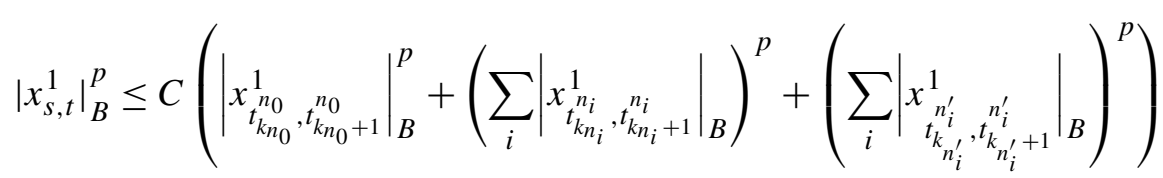

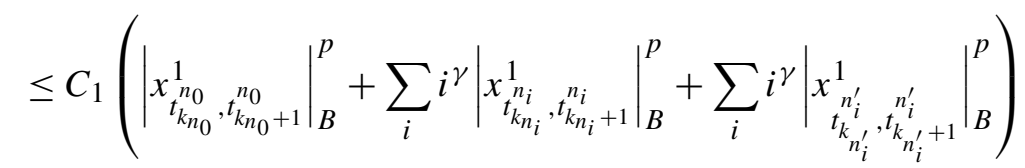

for some constant $C_{1}>0$ only depending on $p$ and $\gamma$. Now let $D=\left\{0=t_{0}<t_{1}<\right.$ $\left.\cdots<t_{r}=1\right\}$ be any finite partition of $[0,1]$. Apply (4.3) to each interval $\left[t_{l-1}, t_{l}\right]$ of this partition. Summing over $l$ we get that

$$
\sum_{l}\left|x_{t_{l-1}, t_{l}}^{1}\right|_{B}^{p} \leq C_{1} \sum_{n=1}^{\infty} n^{\gamma} \sum_{k=1}^{2^{n}}\left|x_{t_{k-1}^{n}, t_{k}^{n}}^{1}\right|_{B}^{p},
$$

where we have used the facts that for different intervals $\left[t_{l-1}, t_{l}\right]$ of the partition $D$, the corresponding dyadic intervals $\left[t_{k-1}^{n}, t_{k}^{n}\right]$ of the preceding construction that 
appear in (4.3) are different, and that the same index $n_{i}$ can only appear at most three times. Since the above upper bound is independent of the partition $D$, we therefore get the conclusion concerning the first level path $x^{1}$.

Next we prove the inequality of the lemma for $x^{2}$. Again, since $\mathbf{x}$ is multiplicative (4.1),

$$
x_{s, t}^{2}=\sum_{i} x_{s_{i-1}, s_{i}}^{2}+\sum_{i<j} x_{s_{i-1}, s_{i}}^{1} \otimes x_{s_{j-1}, s_{j}}^{1},
$$

so that

$$
\begin{aligned}
\left|x_{s, t}^{2}\right|_{B \otimes B}^{p / 2} & \leq 2^{p / 2-1}\left|\sum_{i} x_{s_{i-1}, s_{i}}^{2}\right|_{B \otimes B}^{p / 2}+2^{p / 2-1}\left|\sum_{i<j} x_{s_{i-1}, s_{i}}^{1} \otimes x_{s_{j-1}, s_{j}}^{1}\right|_{B \otimes B}^{p / 2} \\
& \leq 2^{p / 2-1}\left|\sum_{i} x_{s_{i-1}, s_{i}}^{2}\right|_{B \otimes B}^{p / 2}+2^{p / 2-1}\left(\sum_{i}\left|x_{s_{i-1}, s_{i}}^{1}\right|_{B}\right)^{p} .
\end{aligned}
$$

Then by (4.4) and decomposition (4.3) applied similarly to $x^{2}$, we conclude that for some constant $C_{2}>0$,

$$
\sum_{l}\left|x_{t_{l-1}, t_{l}}^{2}\right|_{B \otimes B}^{p / 2} \leq C_{2} \sum_{n=1}^{\infty} n^{\gamma} \sum_{k=1}^{2^{n}}\left(\left|x_{t_{k-1}^{n}, t_{k}^{n}}^{1}\right|_{B}^{p}+\left|x_{t_{k-1}^{n}, t_{k}^{n}}^{2}\right|_{B \otimes B}^{p / 2}\right) .
$$

The lemma is established.

Note that by repeating the argument, we may prove the same result for any level path $x^{i}$, with however some further work (that is not needed here).

As a simple corollary to Lemma 2 and (3.2), we may already state a first consequence for $B$-valued Wiener processes.

COROLlary 3. Let $X=\left(X_{t}\right)_{t>0}$ be a Wiener process with values in a separable Banach space $B$ generated by a Gaussian measure $\mu$. Then, for any $p>2, X$ has almost surely finite $p$-variation on any finite interval. More precisely, if $X_{s, t}^{1}=X_{t}-X_{s}$, we have

$$
\mathbf{E}\left(\sup _{D} \sum_{l}\left|X_{t_{l-1}, t_{l}}^{1}\right|_{B}^{p}\right)<\infty
$$

and therefore

$$
\sup _{D} \sum_{l}\left|X_{t_{l-1}, t_{l}}^{1}\right|_{B}^{p}<\infty \quad \text { almost surely, }
$$

where D runs over all finite partitions on any fixed interval. 
Continuity in $p$-variations may be controlled by the same means. Let $\mathbf{x}$ and $\mathbf{y}$ be two multiplicative functionals in $T^{(2)}(B)$. We may apply the above lemma to the difference $x_{s, t}^{1}-y_{s, t}^{1}$ to get

$$
\sup _{D} \sum_{l}\left|x_{t_{l-1}, t_{l}}^{1}-y_{t_{l-1}, t_{l}}^{1}\right|_{B}^{p} \leq C \sum_{n=1}^{\infty} n^{\gamma} \sum_{k=1}^{2^{n}}\left|x_{t_{k-1}^{n}, t_{k}^{n}}^{1}-y_{t_{k-1}^{n}, t_{k}^{n}}^{1}\right|_{B}^{p} .
$$

The second level $x_{s, t}^{2}-y_{s, t}^{2}$ requires further work.

LEMMA 3. For any $p>2$ and $\gamma>p / 2-1$, there is a constant $C_{2}$ such that

$$
\begin{aligned}
\sup _{D} \sum_{l}\left|x_{t_{l-1}, t_{l}}^{2}-y_{t_{l-1}, t_{l}}^{2}\right|_{B \otimes B}^{p / 2} & \\
\leq & C_{2}\left(\sum_{n=1}^{\infty} n^{\gamma} \sum_{k=1}^{2^{n}}\left(\left|x_{t_{k-1}^{n}, t_{k}^{n}}^{1}-y_{t_{k-1}^{n}, t_{k}^{n}}^{1}\right|_{B}^{p}\right)\right)^{1 / 2} \\
& \times\left(\sum_{n=1}^{\infty} n^{\gamma} \sum_{k=1}^{2^{n}}\left(\left|x_{t_{k-1}^{n}, t_{k}^{n}}^{1}\right|_{B}^{p}+\left|y_{t_{k-1}^{n}, t_{k}^{n}}^{1}\right|_{B}^{p}\right)\right)^{1 / 2} \\
+ & C_{2} \sum_{n=1}^{\infty} n^{\gamma} \sum_{k=1}^{2^{n}}\left|x_{t_{k-1}^{n}, t_{k}^{n}}^{2}-y_{t_{k-1}^{n}, t_{k}^{n}}^{2}\right|_{B \otimes B}^{p / 2} .
\end{aligned}
$$

ProOF. First note that by (4.5) and the notation of Lemma 2,

$$
\begin{aligned}
x_{s, t}^{2}-y_{s, t}^{2}= & \sum_{i}\left(x_{s_{i-1}, s_{i}}^{2}-y_{s_{i-1}, s_{i}}^{2}\right) \\
& +\sum_{i<j}\left(x_{s_{i-1}, s_{i}}^{1}-y_{s_{i-1}, s_{i}}^{1}\right) \otimes x_{s_{j-1}, s_{j}}^{1} \\
& +\sum_{i<j} y_{s_{i-1}, s_{i}}^{1} \otimes\left(x_{s_{j-1}, s_{j}}^{1}-y_{s_{j-1}, s_{j}}^{1}\right) .
\end{aligned}
$$

The first term on the right-hand side of (4.7) is handled as in the proof of Lemma 2 to give rise to the last term on the right-hand side of the inequality of the lemma. By symmetry, it is enough to deal with one of the remaining terms on the right-hand side of (4.7). By the triangle inequality,

$$
\begin{aligned}
& \mid \sum_{i<j}(\left.x_{s_{i-1}, s_{i}}^{1}-y_{s_{i-1}, s_{i}}^{1}\right)\left.\otimes x_{s_{j-1}, s_{j}}^{1}\right|_{B \otimes B} \\
& \quad \leq\left(\sum_{i}\left|x_{s_{i-1}, s_{i}}^{1}-y_{s_{i-1}, s_{i}}^{1}\right|_{B}\right)\left(\sum_{j}\left|x_{s_{j-1}, s_{j}}^{1}\right|_{B}\right)
\end{aligned}
$$


and by $(4.2)$

$$
\begin{aligned}
& \left|\sum_{i<j}\left(x_{s_{i-1}, s_{i}}^{1}-y_{s_{i-1}, s_{i}}^{1}\right) \otimes x_{s_{j-1}, s_{j}}^{1}\right|_{B \otimes B}^{p / 2} \\
& \quad \leq C\left(\sum_{i} i^{\gamma}\left|x_{s_{i-1}, s_{i}}^{1}-y_{s_{i-1}, s_{i}}^{1}\right|_{B}^{p}\right)^{1 / 2}\left(\sum_{j} j^{\gamma}\left|x_{s_{j-1}, s_{j}}^{1}\right|_{B}^{p}\right)^{1 / 2} .
\end{aligned}
$$

We may then conclude as in the proof of Lemma 2. Summing over all intervals $\left[t_{l-1}, t_{l}\right]$ of a partition $D$, and using the Cauchy-Schwarz inequality,

$$
\begin{aligned}
\sum_{l} \mid & \left.\sum_{i<j}\left(x_{s_{i-1}, s_{i}}^{1}-y_{s_{i-1}, s_{i}}^{1}\right) \otimes x_{s_{j-1}, s_{j}}^{1}\right|_{B \otimes B} ^{p / 2} \\
\leq & \left(\sum_{n=1}^{\infty} n^{\gamma} \sum_{k=1}^{2^{n}}\left(\left|x_{t_{k-1}^{n}, t_{k}^{n}}^{1}-y_{t_{k-1}^{n}, t_{k}^{n}}^{1}\right|_{B}^{p}\right)\right)^{1 / 2} \\
& \times\left(\sum_{n=1}^{\infty} n^{\gamma} \sum_{k=1}^{2^{n}}\left(\left|x_{t_{k-1}^{n}, t_{k}^{n}}^{1}\right|_{B}^{p}+\left|y_{t_{k-1}^{n}, t_{k}^{n}}^{1}\right|_{B}^{p}\right)\right)^{1 / 2} .
\end{aligned}
$$

The proof is complete.

5. Geometric rough paths. In this section we construct a geometric rough path canonically associated to a ( $B$-valued) Wiener process. That is, following Lévy's construction of the Brownian area, we prove the dyadic polygonal approximations form a Cauchy sequence. Let thus $X=\left(X_{t}\right)_{t \geq 0}$ be a Wiener process on a probability space $(\Omega, \mathcal{F}, \mathbf{P})$ generated by a Gaussian measure $\mu$ on a Banach space $B$. The general procedure we follow is similar to the classical (real-valued) case via dyadic approximation. However, while (as in Corollary 3 ) property (3.2) will be enough to handle the first level paths, the second level leading to the Lévy area requires a deeper analysis of tensor norm properties.

5.1. The first level paths. Let us first introduce some further notation. For $n \in \mathbf{N}$ and $k=0,1, \ldots, 2^{n}$, let $\Delta_{k}^{n} X=X_{t_{k}^{n}}-X_{t_{k-1}^{n}}\left(=X_{t_{k-1}^{n}, t_{k}^{n}}^{1}\right)$ be the increment of $X$ over interval $\left[t_{k-1}^{n}, t_{k}^{n}\right]$. Let, for any $m \geq 0$,

$$
X(m)_{t}=X_{t_{l-1}^{m}}+2^{m}\left(t-t_{l-1}^{m}\right) \Delta_{l}^{m} X \quad \text { if } t_{l-1}^{m} \leq t \leq t_{l}^{m}
$$

be the continuous, piecewise linear path that coincides with $X$ at the dyadic points $k / 2^{m}$ for $k=0,1, \ldots, 2^{m}$. We then build a geometric rough path $X(m)$ in $T^{(2)}(B)$ from $X(m)$ by taking its iterated integrals. That is,

$$
X(m)_{s, t}^{1}=X(m)_{t}-X(m)_{s}
$$


while

$$
\begin{aligned}
X(m)_{s, t}^{2} & =\int_{s<t_{1}<t_{2}<t} d X(m)_{t_{1}} \otimes d X(m)_{t_{2}} \\
& =\int_{s}^{t}\left(X(m)_{u}-X(m)_{s}\right) \otimes d X(m)_{u} .
\end{aligned}
$$

Surely the dyadic polygonal approximations are of bounded variation for each $m$, and hence have finite $p$-variation for all $p \geq 1$. Therefore, by definition, each $X(m)$ is a geometric rough path with finite $p$-variation for any $2<p<3$.

The following proposition will settle the case of the first level paths (5.1).

PROPOSITION 1. For any $\gamma>0$,

$$
\sup _{m} \mathbf{E}\left(\sum_{n=1}^{\infty} n^{\gamma} \sum_{k=1}^{2^{n}} \mid X(m)_{t_{k-1}^{n},\left.t_{k}^{n}\right|_{B} ^{p}}^{1}<\infty .\right.
$$

PROOF. If $n \leq m$, then $X(m)_{t_{k}^{n}}=X_{t_{k}^{n}}$ so that in this case $X(m)_{t_{k-1}^{n}, t_{k}^{n}}^{1}=\Delta_{k}^{n} X$ for every $k=1, \ldots, 2^{n}$, and

$$
\sum_{k=1}^{2^{n}}\left|X(m)_{t_{k-1}^{n}, t_{k}^{n}}^{1}\right|_{B}^{p}=\sum_{k=1}^{2^{n}}\left|\Delta_{k}^{n} X\right|_{B}^{p} .
$$

If $n>m$ and $1 \leq k \leq 2^{n}$, then we can choose a unique $l$ such that

$$
t_{l-1}^{m} \leq t_{k-1}^{n}<t_{k}^{n}<t_{l}^{m} .
$$

Therefore

$$
X(m)_{t_{j}^{n}}=X_{t_{l-1}^{m}}+2^{m}\left(t_{j}^{n}-t_{l-1}^{m}\right) \Delta_{l}^{m} X, \quad j=k, k-1 .
$$

Hence if $n>m$ and $t_{l-1}^{m} \leq t_{k-1}^{n}<t_{l}^{m}$,

$$
X(m)_{t_{k-1}^{n}, t_{k}^{n}}^{1}=2^{m-n} \Delta_{l}^{m} X .
$$

It follows that

$$
\begin{aligned}
\sum_{k=1}^{2^{n}}\left|X(m)_{t_{k-1}^{n}, t_{k}^{n}}^{1}\right|_{B}^{p} & =\sum_{l=1}^{2^{m}} \sum_{t_{l-1}^{m} \leq t_{k-1}^{n}<t_{l}^{m}}\left|2^{m-n} \Delta_{l}^{m} X\right|_{B}^{p} \\
& =\left(2^{m-n}\right)^{p-1} \sum_{l=1}^{2^{m}}\left|\Delta_{l}^{m} X\right|_{B}^{p} .
\end{aligned}
$$


We can then decompose

$$
\begin{aligned}
\mathbf{E}\left(\sum_{n=1}^{\infty} n^{\gamma} \sum_{k=1}^{2^{n}}\left|X(m)_{t_{k-1}^{n}, t_{k}^{n}}^{1}\right|_{B}^{p}\right) \\
=\mathbf{E}\left(\sum_{n=1}^{m} n^{\gamma} \sum_{k=1}^{2^{n}} \mid X(m)_{t_{k-1}^{n},\left.t_{k}^{n}\right|_{B} ^{p}}^{1}+\mathbf{E}\left(\sum_{n=m+1}^{\infty} n^{\gamma} \sum_{k=1}^{2^{n}}\left|X(m)_{t_{k-1}^{n}, t_{k}^{n}}^{1}\right|_{B}^{p}\right)\right. \\
=\sum_{n=1}^{m} n^{\gamma} \sum_{k=1}^{2^{n}} \mathbf{E}\left(\left|\Delta_{k}^{n} X\right|_{B}^{p}\right)+\sum_{n=m+1}^{\infty} n^{\gamma}\left(2^{m-n}\right)^{p-1} \sum_{l=1}^{2^{m}} \mathbf{E}\left(\left|\Delta_{l}^{m} X\right|_{B}^{p}\right) \\
\leq C \sum_{n=1}^{\infty} n^{\gamma} 2^{-n(p-2) / 2}<\infty
\end{aligned}
$$

where we used (3.2) in the last step. The proof of the proposition is thus complete.

COROLlary 4. For any $p>2$,

$$
\mathbf{E}\left(\sup _{m} \sup _{D} \sum_{l}\left|X(m)_{t_{l-1}, t_{l}}^{1}\right|_{B}^{p}\right)<\infty
$$

and therefore

$$
\sup _{m} \sup _{D} \sum_{l}\left|X(m)_{t_{l-1}, t_{l}}^{1}\right|_{B}^{p}<\infty \quad \text { almost surely. }
$$

PROOF. We first observe that, for any $n \in \mathbf{N}$,

$$
m \mapsto \sum_{k=1}^{2^{n}}\left|X(m)_{t_{k-1}^{n}, t_{k}^{n}}^{1}\right|_{B}^{p}
$$

is increasing. From the proof of Proposition 1, it is enough to consider the case $n>m$ for which we have seen that

$$
\sum_{k=1}^{2^{n}}\left|X(m)_{t_{k-1}^{n}, t_{k}^{n}}^{1}\right|_{B}^{p}=\left(2^{m-n}\right)^{p-1} \sum_{l=1}^{2^{m}}\left|\Delta_{l}^{m} X\right|_{B}^{p} .
$$

But the latter is increasing in $m$ since $\Delta_{l}^{m} X=\Delta_{2 l}^{m+1} X+\Delta_{2 l-1}^{m+1} X$ and

$$
\begin{aligned}
\left(2^{m}\right)^{p-1} \sum_{l=1}^{2^{m}}\left|\Delta_{l}^{m} X\right|_{B}^{p} & \leq\left(2^{m+1}\right)^{p-1} \sum_{l=1}^{2^{m}}\left|\Delta_{2 l}^{m+1} X+\Delta_{2 l-1}^{m+1} X\right|_{B}^{p} \\
& \leq\left(2^{m+1}\right)^{p-1} \sum_{l=1}^{2^{m}}\left(\left|\Delta_{2 l}^{m+1} X\right|_{B}^{p}+\left|\Delta_{2 l-1}^{m+1} X\right|_{B}^{p}\right)
\end{aligned}
$$




$$
=\left(2^{m+1}\right)^{p-1} \sum_{l=1}^{2^{m+1}}\left|\Delta_{l}^{m+1} X\right|_{B}^{p} .
$$

Now, as a consequence of Lemma 2,

$$
\begin{aligned}
\mathbf{E}\left(\sup _{m} \sup _{D} \sum_{l}\left|X(m)_{t_{l-1}, t_{l}}^{1}\right|_{B}^{p}\right) & \leq C_{1} \mathbf{E}\left(\sup _{m} \sum_{n=1}^{\infty} n^{\gamma} \sum_{k=1}^{2^{n}} \mid X(m)_{t_{k-1}^{n},\left.t_{k}^{n}\right|_{B} ^{p}}^{1}\right. \\
& \leq C_{1} \lim _{m \rightarrow \infty} \mathbf{E}\left(\sum_{n=1}^{\infty} n^{\gamma} \sum_{k=1}^{2^{n}}\left|X(m)_{t_{k-1}^{n}, t_{k}^{n}}^{1}\right|_{B}^{p}\right) .
\end{aligned}
$$

The conclusion follows from Proposition 1.

The convergence in $p$-variation of the dyadic approximations to the Brownian paths easily follows from the preceding argument. We already noticed that $X(m)_{t_{k-1}^{n}, t_{k}^{n}}^{1}=\Delta_{k}^{n} X$ for $n \leq m$. If $n>m$, we have

$$
\left|X(m)_{t_{k-1}^{n}, t_{k}^{n}}^{1}-\Delta_{k}^{n} X\right|_{B}^{p} \leq 2^{p-1}\left|X(m)_{t_{k-1}^{n}, t_{k}^{n}}^{1}\right|_{B}^{p}+2^{p-1}\left|\Delta_{k}^{n} X\right|_{B}^{p} .
$$

Therefore, as in the proof of Proposition 1,

$$
\begin{aligned}
& \mathbf{E}\left(\sum_{n=1}^{\infty} n^{\gamma} \sum_{k=1}^{2^{n}}\left|X(m)_{t_{k-1}^{n}, t_{k}^{n}}^{1}-\Delta_{k}^{n} X\right|_{B}^{p}\right) \\
& \quad=\mathbf{E}\left(\sum_{n=m+1}^{\infty} n^{\gamma} \sum_{k=1}^{2^{n}}\left|X(m)_{t_{k-1}^{n}, t_{k}^{n}}^{1}-\Delta_{k}^{n} X\right|_{B}^{p}\right) \\
& \quad \leq C \sum_{n=m+1}^{\infty} n^{\gamma} 2^{-n(p-2) / 2} \\
& \leq C 2^{-m(p-2) / 4}
\end{aligned}
$$

(for example). By means of (4.6), we have thus proved the following result.

TheOREM 2. Set $X_{s, t}^{1}=X_{t}-X_{s}, s<t$. For any $p>2$,

$$
\lim _{m \rightarrow \infty} \sup _{D} \sum_{l}\left|X(m)_{t_{l-1}, t_{l}}^{1}-X_{t_{l-1}, t_{l}}^{1}\right|_{B}^{p}=0 \quad \text { almost surely. }
$$

In other words, the dyadic approximations $X^{1}(m)$ converge almost surely in $p$-variations $(p>2)$ to the first level path $X^{1}$. 
5.2. Constructing Lévy area process. Next we consider the second level paths $X(m)_{s, t}^{2}$ of (5.2). Our aim will be to show that the dyadic approximations $X^{2}(m)$ converge almost surely in $p / 2$-variation thus defining the Lévy area process $X^{2}$, and together with the preceding subsection a geometric rough path associated to the Wiener process on a Banach space $B$. The construction will depend on the choice of the tensor norm on $B \otimes B$ and/or the underlying Gaussian distribution $\mu$. We fix to start with a tensor norm $|\cdot|_{B \otimes B}$ on $B \otimes B$.

A first direct calculation shows that when $n>m$,

$$
X(m)_{t_{k-1}^{n}, t_{k}^{n}}^{2}=\frac{1}{2}\left(2^{m-n}\right)^{2} \Delta_{l}^{m} X \otimes \Delta_{l}^{m} X
$$

where $l$ is the unique nonnegative integer such that

$$
t_{l-1}^{m} \leq t_{k-1}^{n}<t_{k}^{n}<t_{l}^{m} .
$$

It is then a simple matter to show by (3.2) as in Section 5.1 that for some constant $C>0$ only depending on $p>2$, and all $n>m$,

$$
\sum_{k=1}^{2^{n}} \mathbf{E}\left(\left|X(m)_{t_{k-1}^{n}, t_{k}^{n}}^{2}\right|_{B \otimes B}^{p / 2}\right) \leq C 2^{-n(p-1)} 2^{m p / 2} .
$$

If $n \leq m$, it is easy to check that $X(m)_{t_{k-1}^{n}, t_{k}^{n}}^{2}, k=1, \ldots, 2^{n}$, splits into a symmetric and an antisymmetric part as

$$
\begin{aligned}
X(m)_{t_{k-1}^{n}, t_{k}^{n}}^{2}= & \frac{1}{2} \Delta_{k}^{n} X \otimes \Delta_{k}^{n} X \\
& +\frac{1}{2} \sum_{r, l=2^{m-n}(k-1)+1, r<l}^{2^{m-n} k}\left(\Delta_{r}^{m} X \otimes \Delta_{l}^{m} X-\Delta_{l}^{m} X \otimes \Delta_{r}^{m} X\right) .
\end{aligned}
$$

Therefore,

$$
\begin{aligned}
& X(m+1)_{t_{k-1}^{n}, t_{k}^{n}-X(m)_{t_{k-1}^{n}, t_{k}^{n}}^{2}} \\
& \quad=\frac{1}{2} \sum_{l=2^{m-n}(k-1)+1}^{2^{m-n} k}\left(\Delta_{2 l-1}^{m+1} X \otimes \Delta_{2 l}^{m+1} X-\Delta_{2 l}^{m+1} X \otimes \Delta_{2 l-1}^{m+1} X\right) .
\end{aligned}
$$

At this point, the issue becomes more delicate, and requires a careful analysis of tensor norms on $B \otimes B$ to handle

$$
\left|\sum_{l=2^{m-n}(k-1)+1}^{2^{m-n} k} \Delta_{2 l}^{m+1} X \otimes \Delta_{2 l-1}^{m+1} X\right|_{B \otimes B} .
$$

The following definition will allow us to treat families of interesting examples in the next section. 
DEFinition 1. Let $B$ be a Banach space and let $\mu$ be a centered Gaussian distribution on the Borel sets of $B$. Let $|\cdot|_{B \otimes B}$ be a Banach tensor norm on $B \otimes B$. We say that the pair $\left(|\cdot|_{B \otimes B}, \mu\right)$ is exact if there exist $\left(\frac{1}{2} \leq\right) \alpha<1$ and a constant $C>0$, such that for any sequence $\left\{G_{l}, \widetilde{G}_{l}\right\}_{l \geq 1}$ of independent $B$-valued Gaussian random variables with identical distribution $\mu$, and any $N \geq 1$,

$$
\mathbf{E}\left(\left|\sum_{l=1}^{N} G_{l} \otimes \widetilde{G}_{l}\right|_{B \otimes B}\right) \leq C N^{\alpha} .
$$

We also say below that a given tensor norm is exact with respect to a given Gaussian distribution.

Anticipating the next section, let us mention that by elementary variance bounds every tensor norm and Gaussian distribution is exact (with $\alpha=\frac{1}{2}$ ) whenever $B$ is finite dimensional. Actually, the sequence $\left\{G_{l} \otimes \widetilde{G}_{l}\right\}$ satisfies in this case a central limit theorem in $B \otimes B$. Due to the delicate structure of tensor norms in the infinite dimensional case (even when $B$ is nice), it does not seem straightforward to check exactness in the sense of the preceding definition. At the level of the law of large numbers (cf. [19] for basics on limit theorems in Banach spaces), for any tensor norm and any distribution $\mu$,

$$
\mathbf{E}\left(\left|\sum_{l=1}^{N} G_{l} \otimes \widetilde{G}_{l}\right|_{B \otimes B}\right)=o(N)
$$

as $N \rightarrow \infty$ [to be proved below in (6.2)]. This is however the best that can hold in general: as was pointed out to us by Kwapien and Pisier, one may construct Gaussian measures on Hilbert space such that the rate $o(N)$ in (5.6) for the projective norm is as slow as one wishes it. In Section 5 we present both examples of tensor norms and of Gaussian measures that are exact. It is convenient to notice that by the equivalence of moments (3.1) of Gaussian random vectors $|\cdot|_{B \otimes B}$ is exact with parameter $\alpha<1$ if and only if for some, or all, $1 \leq q<\infty$,

$$
\mathbf{E}\left(\left|\sum_{l=1}^{N} G_{l} \otimes \widetilde{G}_{l}\right|_{B \otimes B}^{q}\right) \leq C N^{\alpha q}
$$

for some constant $C>0$ and all $N \geq 1$. Indeed, for a fixed realization of $\left\{G_{1}, \ldots, G_{N}\right\}, \sum_{l=1}^{N} G_{l} \otimes \widetilde{G}_{l}$ is a Gaussian random vector in $B \otimes B$ to which one may apply (3.1). Apply then again (3.1) to the norm

$$
\left(\widetilde{\mathbf{E}}\left(\left|\sum_{l=1}^{N} G_{l} \otimes \widetilde{G}_{l}\right|_{B \otimes B}^{q}\right)\right)^{1 / q}
$$

of the vector $\left\{G_{1}, \ldots, G_{N}\right\}$ (where $\widetilde{\mathbf{E}}$ is partial integration with respect to $\left.\left\{\widetilde{G}_{1}, \ldots, \widetilde{G}_{N}\right\}\right)$. 
Now, assume in the following that the tensor norm $|\cdot|_{B \otimes B}$ is exact with respect to the Gaussian measure $\mu$. Then, since for any $k$, the random variables

$$
\left\{\sqrt{2^{m+1}} \Delta_{2 l}^{m+1} X, \sqrt{2^{m+1}} \Delta_{2 l-1}^{m+1} X, l=2^{m-n}(k-1)+1, \ldots, 2^{m-n} k\right\},
$$

are independent with identical distribution $\mu$, for any $m \geq n$,

$$
\begin{aligned}
& \mathbf{E}\left(\left.\left.\right|_{l=2^{m-n}(k-1)+1} \Delta_{2 l}^{m+1} X \otimes \Delta_{2 l-1}^{m+1} X\right|_{B \otimes B} ^{p / 2}\right) \\
& \quad=2^{-(m+1) p / 2} \mathbf{E}\left(\left|\sum_{l=1}^{2^{m-n}} \sqrt{2^{m+1}} \Delta_{2 l}^{m+1} X \otimes \sqrt{2^{m+1}} \Delta_{2 l-1}^{m+1} X\right|_{B \otimes B}^{p / 2}\right) \\
& \quad \leq C 2^{-(m+1) p / 2}\left(2^{m-n}\right)^{\alpha p / 2} \\
& \leq C 2^{-m(1-\alpha) p / 2} 2^{-n \alpha p / 2} .
\end{aligned}
$$

Therefore, by (5.5),

$$
\sum_{k=1}^{2^{n}} \mathbf{E}\left(\left|X(m+1)_{t_{k-1}^{n}, t_{k}^{n}}^{2}-X(m)_{t_{k-1}^{n}, t_{k}^{n}}^{2}\right|_{B \otimes B}^{p / 2}\right) \leq C 2^{-m(1-\alpha) p / 2} 2^{-n(\alpha p / 2-1)}
$$

Now we make use of Lemma 3. Together with Proposition 1 and (5.3), it follows from (5.4) and the preceding that since $\alpha<1$,

$$
\begin{aligned}
& \mathbf{E}\left(\sup _{D} \sum_{l}\left|X(m+1)_{t_{l-1}, t_{l}}^{2}-X(m)_{t_{l-1}, t_{l}}^{2}\right|_{B \otimes B}^{p / 2}\right) \\
& \quad \leq C\left(2^{-m(p-2) / 8}+\sum_{n>m} n^{\gamma} 2^{-n(p-1)} 2^{m p / 2}+\sum_{n \leq m} n^{\gamma} 2^{-m(1-\alpha) p / 2} 2^{-n(\alpha p / 2-1)}\right) \\
& \quad \leq C\left(2^{-m(p-2) / 8}+m^{\gamma} 2^{-n(p / 2-1)}+2^{-m(1-\alpha) p / 2}\right) \\
& \quad \leq C\left(2^{-m(p-2) / 8}+2^{-m(1-\alpha) p / 2}\right)
\end{aligned}
$$

for some constant $C$ possibly changing from line to line and depending only on $p>2, \gamma>p / 2-1$ and $\alpha<1$ (where we distinguish according as $\alpha p \leq 2$ and $\alpha p>2)$. Therefore,

$$
\mathbf{E}\left(\sum_{m=1}^{\infty}\left(\sup _{D} \sum_{l}\left|X(m+1)_{t_{l-1}, t_{l}}^{2}-X(m)_{t_{l-1}, t_{l}}^{2}\right|_{B \otimes B}^{p / 2}\right)^{2 / p}\right)<\infty
$$

so that in particular,

$$
\sum_{m=1}^{\infty}\left(\sup _{D} \sum_{l}\left|X(m+1)_{t_{l-1}, t_{l}}^{2}-X(m)_{t_{l-1}, t_{l}}^{2}\right|_{B \otimes B}^{p / 2}\right)^{2 / p}<\infty
$$


almost surely. It follows that $\left(X(m)^{2}\right)$ is almost surely a Cauchy sequence in $p / 2$ variation norm so that, for some element $X$ in $T^{(2)}(B)$,

$$
X(m)^{2} \rightarrow X^{2} \quad \text { almost surely }
$$

in $p / 2$-variation as $m \rightarrow \infty$. Existence of the second level path $X^{2}$ (Lévy area) is established in this way. Together with Theorem 2, we have thus proved the following.

THEOREM 3. Let $X=\left(X_{t}\right)_{t \geq 0}$ be a B-valued Wiener process generated by $a$ Gaussian measure $\mu$ on $B$. Equip the tensor product $B \otimes B$ with a tensor norm $|\cdot|_{B \otimes B}$ such that the pair $\left(|\cdot|_{B \otimes B}, \mu\right)$ is exact. Then, for this topology on $B \otimes B$, the dyadic approximations $\mathbf{X}(m)=\left(1, X(m)^{1}, X(m)^{2}\right)$ converge to geometric rough paths $\mathbf{X}=\left(1, X^{1}, X^{2}\right)$ in $p$-variation distance almost surely for any $2<p<3$. Moreover, $X_{s, t}^{1}=X_{t}-X_{s}$ for any $s<t$.

We call $\mathbf{X}_{s, t}=\left(1, X_{s, t}^{1}, X_{s, t}^{2}\right), 0 \leq s<t \leq 1$, the canonical geometric rough path associated to the Wiener process $X$ for the tensor norm $|\cdot|_{B \otimes B}$. In this case we also say the canonical Lévy area process of $X$ exists in the tensor product $\left(B \otimes B,|\cdot|_{B \otimes B}\right)$.

It might be possible to improve the exactness hypothesis in the preceding theorem by a careful analysis of the decay in (5.6) that would be necessary in the proof. We however believe that this does not lead to any really significant better condition. In any case, (5.6) alone would not be enough.

6. Examples of applications. In this section, we concentrate on examples of Gaussian measures and tensor norms which are exact in the sense of Definition 1. We first show that the injective tensor norm (the smallest among tensor norms) is exact (with $\alpha=\frac{1}{2}$ ) for any Gaussian measure $\mu$ on a Banach space $B$. Then we deal with the projective tensor norm (the largest among tensor norms) but for particular classes of Gaussian measures that we describe under increment or metric entropy conditions. Recall that by elementary variance bounds, every tensor norm is exact on a finite dimensional Banach space $B$.

Each element $v$ in the linear tensor product $B \otimes B$ of a Banach space $B$ with itself has a representation

$$
v=\sum_{i=1}^{n} x_{i} \otimes y_{i} \quad \text { for } x_{i} \in B, y_{i} \in B .
$$

We may clearly regard $v$ also as a bilinear form on $B^{*} \times B^{*}$.

The injective tensor norm $|\cdot|_{\vee}$ on $B \otimes B$ is defined as

$$
|v|_{\vee}=\sup \sum_{i=1}^{n}\left\langle x_{i}, \xi\right\rangle\left\langle y_{i}, \eta\right\rangle
$$


where the supremum is running over all elements $\xi$ and $\eta$ in the unit ball $B_{1}^{*}$ of the dual space of $B$. This norm coincides with the norm of $v$ as a bounded bilinear form on $B^{*} \times B^{*}$. The projective norm $|\cdot|_{\wedge}$ is defined by

$$
|v|_{\wedge}=\inf \sum_{i=1}^{n}\left|x_{i}\right|_{B}\left|y_{i}\right|_{B}
$$

where the infinemum is running over all possible representations $v=\sum_{i=1}^{n} x_{i} \otimes y_{i}$. Clearly $|v|_{\vee} \leq|v|_{\wedge}$. The norms $|\cdot|_{\wedge}$ and $|\cdot|_{\vee}$ are respectively the largest and the smallest reasonable norm on $B \otimes B$. Actually, the projective tensor norm is better described in its dual form as

$$
|v|_{\wedge}=\sup \sum_{i=1}^{n}\left\langle x_{i}, \varphi\left(y_{i}\right)\right\rangle
$$

where the supremum is running over all linear forms $\varphi: B \rightarrow B^{*}$ of norm less than or equal to 1 . Each reasonable norm on $B \otimes B$ may be described in this way for some family of linear functionals $\varphi$. The injective norm corresponds to the family of all $\varphi=\xi \otimes \eta, \xi, \eta \in B_{1}^{*}$. The projective (resp. injective) tensor product is the completion of the linear tensor product $B \otimes B$ with respect to $|\cdot|_{\vee}\left(\right.$ resp. $\left.|\cdot|_{\wedge}\right)$.

We mention furthermore that the projective tensor product of $L^{1}(\Sigma)$ is $L^{1}(\Sigma$ $\times \Sigma)$ while the injective tensor product of $L^{\infty}(\Sigma)$ is $L^{\infty}(\Sigma \times \Sigma)$. For $1<$ $p<\infty, L^{p}(\Sigma \times \Sigma)$ provides an intermediate scale between the projective and injective tensors of $L^{p}$-spaces. In particular, for $p=2$, the Hilbert tensor product $L^{2}(\Sigma \times \Sigma)$ is again Hilbert. It is easily seen that the $L^{p}(\Sigma \times \Sigma), 1 \leq p<\infty$, tensor product is exact (with $\alpha=\frac{1}{2}$ ) for any Gaussian measure. Namely,

$$
\begin{aligned}
& \mathbf{E}\left(\left|\sum_{l=1}^{N} G_{l} \otimes \widetilde{G}_{l}\right|_{L^{p}(\Sigma \times \Sigma)}^{p}\right) \\
& \quad=\iint_{\Sigma \times \Sigma} \mathbf{E}\left(\left|\sum_{l=1}^{N} G_{l}(\sigma) \widetilde{G}_{l}\left(\sigma^{\prime}\right)\right|^{p} d \sigma d \sigma^{\prime}\right) \\
& \quad \leq C_{p} \sqrt{N} \iint_{\Sigma \times \Sigma}\left(\mathbf{E}\left(\left|G_{1}(\sigma)\right|^{2}\right)\right)^{p / 2}\left(\mathbf{E}\left(\left|\widetilde{G}_{1}(\sigma)\right|^{2}\right)\right)^{p / 2} d \sigma d \sigma^{\prime} \\
& \quad \leq C_{p} \sqrt{N} \mathbf{E}\left(\left|G_{1}\right|_{L^{p}(\Sigma)}^{p}\right)^{2}
\end{aligned}
$$

where we used (5.7) (in the real case). In particular, the projective tensor product is exact with respect to any Gaussian measure on $L^{1}$ spaces. Our next result extends the preceding to the case $p=\infty$.

6.1. Injective tensor products. The following theorem shows that the injective tensor is exact with respect to any Gaussian distribution. Its proof goes back to the argument put forward by Chevet [7] (cf. [19]) in her investigation of Gaussian 
random series in injective tensor spaces and relies on the Gaussian comparison theorems (Theorem 1).

THEOREM 4. Let $(B, H, \mu)$ be an abstract Wiener space. Let $\left\{G_{l} ; \widetilde{G}_{l}\right\}_{l \geq 1}$ be independent centered Gaussian random variables with identical distribution $\mu$. There is an absolute constant $C>0$ such that for every $N \geq 1$,

$$
\mathbf{E}\left(\left|\sum_{l=1}^{N} G_{l} \otimes \widetilde{G}_{l}\right|_{\vee}\right) \leq C \sqrt{N} \mathbf{E}\left(\left|G_{1}\right|_{B}^{2}\right) .
$$

In other words, the injective tensor product is exact (with $\alpha=\frac{1}{2}$ ) with respect to any Gaussian measure.

PROOF. The main idea of the proof is to construct a comparison Gaussian process via Theorem 1. By homogeneity, and for simplicity, we may assume that $\mathbf{E}\left(\left|G_{1}\right|_{B}^{2}\right)=1$. By definition of the injective tensor norm,

$$
\left|\sum_{l=1}^{N} G_{l} \otimes \widetilde{G}_{l}\right|_{V}=\sup _{(\xi, \eta) \in B_{1}^{*} \times B_{1}^{*}} \sum_{l=1}^{N}\left\langle G_{l}, \xi\right\rangle\left\langle\widetilde{G}_{l}, \eta\right\rangle .
$$

Fix a realization $\widetilde{G}_{l}=\tilde{x}_{l}, l=1, \ldots, N$, and consider the Gaussian process $\left(G_{(\xi, \eta)}\right)$ indexed by $B_{1}^{*} \times B_{1}^{*}$ defined by

$$
G_{(\xi, \eta)}=\sum_{l=1}^{N}\left\langle G_{l}, \xi\right\rangle\left\langle\tilde{x}_{l}, \eta\right\rangle
$$

It is easy to see that for $(\xi, \eta),\left(\xi^{\prime}, \eta^{\prime}\right) \in B_{1}^{*} \times B_{1}^{*}$,

$$
\begin{aligned}
& \mathbf{E}\left(\left|G_{(\xi, \eta)}-G_{\left(\xi^{\prime}, \eta^{\prime}\right)}\right|^{2}\right) \\
& \leq 2 \mathbf{E}\left(\left|\sum_{l=1}^{N}\left(\left\langle G_{l}, \xi\right\rangle-\left\langle G_{l}, \xi^{\prime}\right\rangle\right)\left\langle\tilde{x}_{l}, \eta\right\rangle\right|^{2}\right) \\
&+2 \mathbf{E}\left(\left|\sum_{l=1}^{N}\left\langle G_{l}, \xi^{\prime}\right\rangle\left(\left\langle\tilde{x}_{l}, \eta\right\rangle-\left\langle\tilde{x}_{l}, \eta^{\prime}\right\rangle\right)\right|^{2}\right) \\
& \leq 2 \sum_{l=1}^{N} \mathbf{E}\left(\left|\left\langle G_{l}, \xi\right\rangle-\left\langle G_{l}, \xi^{\prime}\right\rangle\right|^{2}\right)\left\langle\tilde{x}_{l}, \eta\right\rangle^{2} \\
&+2 \sum_{l=1}^{N} \mathbf{E}\left(\left\langle G_{l}, \xi^{\prime}\right\rangle^{2}\right)\left|\left\langle\tilde{x}_{l}, \eta\right\rangle-\left\langle\tilde{x}_{l}, \eta^{\prime}\right\rangle\right|^{2} \\
& \leq 2 \sum_{l=1}^{N}\left|\tilde{x}_{l}\right|_{B}^{2} \mathbf{E}\left(\left|\left\langle G_{1}, \xi\right\rangle-\left\langle G_{1}, \xi^{\prime}\right\rangle\right|^{2}\right)+2 \sum_{l=1}^{N}\left|\left\langle\tilde{x}_{l}, \eta\right\rangle-\left\langle\tilde{x}_{l}, \eta^{\prime}\right\rangle\right|^{2}
\end{aligned}
$$


[where we used that $\mathbf{E}\left(\left|G_{1}\right|_{B}^{2}\right)=1$ ]. Consider then the Gaussian process $\left(G_{(\xi, \eta)}^{\prime}\right)$ also indexed by $B_{1}^{*} \times B_{1}^{*}$ defined by

$$
G_{(\xi, \eta)}^{\prime}=\sqrt{2}\left(\sum_{l=1}^{N}\left|\tilde{x}_{l}\right|_{B}^{2}\right)^{1 / 2}\left\langle\xi, G_{1}\right\rangle+\sqrt{2} \sum_{l=1}^{N} g_{l}^{\prime}\left\langle\tilde{x}_{l}, \eta\right\rangle
$$

where $g_{1}^{\prime}, \ldots, g_{N}^{\prime}$ are independent standard normal variables independent of the sequence $\left\{G_{l} ; \widetilde{G}_{l}\right\}_{l \geq 1}$. We thus have that for every $(\xi, \eta),\left(\xi^{\prime}, \eta^{\prime}\right) \in B_{1}^{*} \times B_{1}^{*}$, $d_{G}\left((\xi, \eta),\left(\xi^{\prime}, \eta^{\prime}\right)\right) \leq d_{G^{\prime}}\left((\xi, \eta),\left(\xi^{\prime}, \eta^{\prime}\right)\right)$. Therefore, by Theorem 1 and $(6.1)$,

$$
\mathbf{E}\left(\left|\sum_{l=1}^{N} G_{l} \otimes \tilde{x}_{l}\right|_{\vee}\right) \leq \sqrt{2}\left(\sum_{l=1}^{N}\left|\tilde{x}_{l}\right|_{B}^{2}\right)^{1 / 2}+\sqrt{2} \mathbf{E}\left(\left|\sum_{l=1}^{N} g_{l}^{\prime} \tilde{x}_{l}\right|_{B}\right) .
$$

Integrate now in the variables $\widetilde{G}_{1}, \ldots, \widetilde{G}_{N}$. We get

$$
\begin{aligned}
\mathbf{E}\left(\left|\sum_{l=1}^{N} G_{l} \otimes \widetilde{G}_{l}\right|_{V}\right) & \leq \sqrt{2} \mathbf{E}\left(\left(\sum_{l=1}^{N}\left|\widetilde{G}_{l}\right|_{B}^{2}\right)^{1 / 2}\right)+\sqrt{2} \mathbf{E}\left(\left|\sum_{l=1}^{N} g_{l}^{\prime} \widetilde{G}_{l}\right|_{B}\right) \\
& \leq \sqrt{2 N}+\sqrt{2} \mathbf{E}\left(\left|\sum_{l=1}^{N} g_{l}^{\prime} \widetilde{G}_{l}\right|_{B}\right) .
\end{aligned}
$$

Using the fact that $\sum_{l=1}^{N} g_{l}^{\prime} \widetilde{G}_{l}$ has the same distribution as $\left(\sum_{l=1}^{N} g_{l}^{\prime 2}\right)^{1 / 2} \widetilde{G}_{1}$ conditionally on $\left\{g_{1}^{\prime}, \ldots, g_{N}^{\prime}\right\}$, we get that

$$
\mathbf{E}\left(\left|\sum_{l=1}^{N} G_{l} \otimes \widetilde{G}_{l}\right|_{V}\right) \leq \sqrt{2 N}+\sqrt{2} \mathbf{E}\left(\left(\sum_{l=1}^{N} g_{l}^{\prime 2}\right)^{1 / 2}\right) \leq 2 \sqrt{2 N}
$$

which is the desired result. The proof of Theorem 4 is complete.

The preceding proof of Theorem 4 does not require the $\widetilde{G}_{l}$ 's to be Gaussian. After some modifications, it actually shows similarly that if the $\widetilde{G}_{l}$ 's satisfy the central limit theorem in $B$ (or in some other Banach space), that is, $\frac{1}{\sqrt{N}} \sum_{l=1}^{N} \widetilde{G}_{l}$ converges weakly, and if the $G_{l}$ 's are independent identically Gaussian, then the $G_{l} \otimes \widetilde{G}_{l}$ 's satisfy the central limit theorem in the injective tensor $B \otimes B$, that is, $\frac{1}{\sqrt{N}} \sum_{l=1}^{N} G_{l} \otimes \widetilde{G}_{l}$ converges weakly in $B \otimes B$, a result of possible independent interest. This observation has been noticed in discussions with $\mathrm{X}$. Fernique and the first named author some years ago.

6.2. Finite dimensional approximation. In this section, we rather consider arbitrary tensor norms but restrict to some families of Gaussian measures. We do not try to get the best possible conditions, but only provide some useful criteria. These also cover Wiener measure for Hölder or Besov types norms. As discussed 
in the introduction, the results of Section 5 thus allow us to solve under minimal conditions infinite dimensional SDEs driven by Brownian motions generated by these Gaussian measures.

We first emphasize, as announced after the definition of exactness, that

$$
\mathbf{E}\left(\left|\sum_{l=1}^{N} G_{l} \otimes \widetilde{G}_{l}\right|_{B \otimes B}\right)=o(N)
$$

for any tensor norm and any Gaussian measure $\mu$. Assume for simplicity that $\mathbf{E}\left(\left|G_{1}\right|_{B}^{2}\right)=1$. The principle we develop will be useful later on. Namely, if $\left\{\xi_{i}\right\}$ is an orthonormal basis of $H$, by Lemma 1 ,

$$
\varepsilon(d)=\mathbf{E}\left(\left|\sum_{i=d+1}^{\infty} g_{i} \xi_{i}\right|_{B}\right) \rightarrow 0
$$

as $d \rightarrow \infty$. Set $F=\sum_{i=1}^{d} g_{i} \xi_{i}$, and denote by $F_{l}, \widetilde{F}_{l}, l \geq 1$, independent copies of $F$. Set also $H_{l}=G_{l}-F_{l}, \tilde{H}_{l}=\widetilde{G}_{l}-\widetilde{F}_{l}, l \geq 1$. We may write that

$$
\sum_{l=1}^{N} G_{l} \otimes \widetilde{G}_{l}=\sum_{l=1}^{N} F_{l} \otimes \widetilde{F}_{l}+\sum_{l=1}^{N} F_{l} \otimes \tilde{H}_{l}+\sum_{l=1}^{N} H_{l} \otimes \widetilde{F}_{l}+\sum_{l=1}^{N} H_{l} \otimes \tilde{H}_{l} .
$$

For any tensor norm $|\cdot|_{B \otimes B}$ on $B \otimes B$, by the triangle inequality,

$$
\left|\sum_{l=1}^{N} F_{l} \otimes \tilde{H}_{l}\right|_{B \otimes B} \leq \sum_{l=1}^{N}\left|F_{l}\right|_{B}\left|\tilde{H}_{l}\right|_{B}
$$

so that

$$
\mathbf{E}\left(\left|\sum_{l=1}^{N} F_{l} \otimes \tilde{H}_{l}\right|_{B \otimes B}\right) \leq N \varepsilon(d)
$$

[since, by Jensen's inequality, $\mathbf{E}\left(\left|F_{1}\right|_{B}\right) \leq \mathbf{E}\left(\left|G_{1}\right|_{B}\right) \leq 1$ ]. Similar bounds hold for $\sum_{l=1}^{N} H_{l} \otimes \widetilde{F}_{l}$ and $\sum_{i=1}^{N} H_{l} \otimes \tilde{H}_{l}$. On the other hand, with the obvious notation,

$$
\left|\sum_{l=1}^{N} F_{l} \otimes \widetilde{F}_{l}\right|_{B \otimes B}=\sup \sum_{i, j=1}^{d}\left(\sum_{l=1}^{N} g_{i}^{l} \widetilde{g}_{j}^{l}\right)\left\langle\xi_{i}, \varphi\left(\xi_{j}\right)\right\rangle
$$

where the supremum is running over all, or a part, of linear functionals $\varphi: B \rightarrow B^{*}$ of norm less than or equal to 1 . Therefore, since $\mathbf{E}\left(\left|\sum_{l=1}^{N} g_{i}^{l} \tilde{g}_{j}^{l}\right|\right) \leq \sqrt{N}$, and for every $i,\left|\xi_{i}\right|_{B}^{2} \leq \mathbf{E}\left(\left|G_{1}\right|_{B}^{2}\right)=1$,

$$
\mathbf{E}\left(\left|\sum_{l=1}^{N} F_{l} \otimes \widetilde{F}_{l}\right|_{B \otimes B}\right) \leq d^{2} \sqrt{N}
$$


Therefore,

$$
\mathbf{E}\left(\left|\sum_{l=1}^{N} G_{l} \otimes \widetilde{G}_{l}\right|_{B \otimes B}\right) \leq d^{2} \sqrt{N}+3 \varepsilon(d) N
$$

from which (6.2) immediately follows.

Estimates on $\varepsilon(d)$ that produce exact tensor norms via the interpolation (6.4) are already available for a number of examples such as Brownian motions and sheets (see, e.g., [13] in the study of rates of convergence in Strassen's law of the iterated logarithm). It is shown, for example, in [13] that $\varepsilon(d) \leq C_{p} d^{-1 / 2} \log d$ for Brownian motion in $\mathbf{R}^{p}$. These even apply to smoother norms such as Hölder or Sobolev norms (see, e.g., [27] and the references therein). Actually, the infimum of $\varepsilon(d)$ over all the representations $\sum_{i=1}^{\infty} g_{i} \xi_{i}$ defines the so-called Gaussian approximation numbers and is related to other properties of the underlying Gaussian distribution $\mu$ such as the degree of compactness of the unit ball of $B$ in $H$ or the small ball behavior of $\mu$ (cf., e.g., the recent contribution [21] and the references therein). We do not need however to enter here the general abstract theory, and only present some useful results and techniques.

We briefly illustrate, somewhat more general, some of these results that rely on the Schauder decomposition of continuous functions, similar actually to the approximations used for the rough path construction in the preceding sections. The argument allows us to also deal with some smoother norms such as Hölder norms. Consider $B$ the space $C([0,1])$ of continuous functions on $[0,1]$ with values in $\mathbf{R}^{d}$. For simplicity in the notation, we set $d=1$. Recall the Haar functions $h_{00}$ and $h_{n k}$, $n \geq 0,1 \leq k \leq 2^{n}$, on $[0,1]$, defined by $h_{00} \equiv 1$ and

$$
h_{n k}(t)= \begin{cases}2^{n / 2}, & \text { if }(k-1) / 2^{n} \leq t<\left(k-\frac{1}{2}\right) / 2^{n}, \\ -2^{n / 2}, & \text { if }\left(k-\frac{1}{2}\right) / 2^{n} \leq t<k / 2^{n}, \\ 0, & \text { otherwise. }\end{cases}
$$

The continuous functions $\varphi_{n k}(t)=\int_{0}^{t} h_{n k}(s) d s, 0 \leq t \leq 1$, are called the Schauder functions. Note that $\left|\varphi_{n k}(t)\right| \leq 2^{-(n / 2)-1}$ for every $0 \leq t \leq 1$. The Schauder functions form a basis for $C([0,1])$ : any function $x$ in $C([0,1])$ may be represented as the uniformly convergent series

$$
x(t)=\sum_{n, k} x_{n k} \varphi_{n k}(t), \quad 0 \leq t \leq 1,
$$

where the coefficients $x_{n k}$ are given by $x_{00}=x(0)+x(1)$ and

$$
x_{n k}=2^{k / 2}\left(2 x\left(\frac{k-(1 / 2)}{2^{n}}\right)-x\left(\frac{k}{2^{n}}\right)-x\left(\frac{k-1}{2^{n}}\right)\right) .
$$

Any continuous (centered) Gaussian process $G=(G(t))_{t \in[0,1]}$ may thus be developed along the Schauder basis as

$$
G(t)=\sum_{n, k} G_{n k} \varphi_{n k}(t), \quad 0 \leq t \leq 1,
$$


where the coefficients $G_{n k}$ are (centered) Gaussian real-valued random variables given by $G_{00}=G(0)+G(1)$ and

$$
G_{n k}=2^{n / 2}\left(2 G\left(\frac{k-(1 / 2)}{2^{n}}\right)-G\left(\frac{k}{2^{n}}\right)-G\left(\frac{k-1}{2^{n}}\right)\right) .
$$

When $G$ is Brownian motion on $[0,1]$, the random variables $G_{n k}$ are independent standard normal variables and the Schauder basis is an orthonormal basis of the Cameron-Martin Hilbert space. The preceding may thus be applied together with estimates on $\varepsilon(d)$. What follows goes a little bit beyond since the $G_{n k}$ 's need not anymore be independent.

For every $m \geq 0$, define

$$
F(t)=F^{m}(t)=\sum_{n \leq m, k} G_{n k} \varphi_{n k}(t), \quad 0 \leq t \leq 1 .
$$

Set also $H(t)=H^{m}(t)=G(t)-F(t), t \in T$. We follow the decomposition (6.3) used in the proof of (6.2). If $G_{l}, \widetilde{G}_{l}$, resp. $F_{l}, \widetilde{F}_{l}, H_{l}, \tilde{H}_{l}, l \geq 1$, are independent copies of $G$, resp. $F, H$, we get similarly

$$
\sum_{l=1}^{N} G_{l} \otimes \widetilde{G}_{l}=\sum_{l=1}^{N} F_{l} \otimes \widetilde{F}_{l}+\sum_{l=1}^{N} F_{l} \otimes \tilde{H}_{l}+\sum_{l=1}^{N} H_{l} \otimes \widetilde{F}_{l}+\sum_{i=1}^{N} H_{l} \otimes \tilde{H}_{l} .
$$

Let $|\cdot|_{B \otimes B}$ be any tensor norm on $B \otimes B$. By the triangle inequality,

$$
\mathbf{E}\left(\left|\sum_{l=1}^{N} F_{l} \otimes \tilde{H}_{l}\right|_{B \otimes B}\right) \leq N \mathbf{E}\left(|F|_{B}\right) \mathbf{E}\left(|H|_{B}\right) .
$$

A similar bound holds for $\sum_{l=1}^{N} H_{l} \otimes \widetilde{F}_{l}$ and $\sum_{l=1}^{N} H_{l} \otimes \tilde{H}_{l}$.

Consider now the uniform norm $|\cdot|_{B}$ on $B=C([0,1])$. Clearly,

$$
\mathbf{E}\left(|F|_{B}\right) \leq \mathbf{E}(|G(0)+G(1)|)+\sum_{n=0}^{m} \mathbf{E}\left(\left|\sum_{k=1}^{2^{n}} G_{n k} \varphi_{n k}\right|_{B}\right) .
$$

Since the $\varphi_{n k}, k=1, \ldots, 2^{n}$, have disjoint supports and since $\left|\varphi_{n k}\right|_{B} \leq 2^{-(n / 2)-1}$,

$$
\mathbf{E}\left(|F|_{B}\right) \leq \mathbf{E}(|G(0)+G(1)|)+\sum_{n=0}^{m} 2^{-n / 2} \mathbf{E}\left(\max _{1 \leq k \leq 2^{n}}\left|G_{n k}\right|\right) .
$$

A classical inequality (cf. [19], page 79) on the maximum of a finite number of (centered) Gaussian random variables indicates that, for every $n \geq 0$,

$$
\mathbf{E}\left(\max _{1 \leq k \leq 2^{n}}\left|G_{n k}\right|\right) \leq K(n+1)^{1 / 2} \max _{1 \leq k \leq 2^{n}}\left(\mathbf{E}\left(G_{n k}^{2}\right)\right)^{1 / 2}
$$

where $K>0$ is some numerical constant. 
Assume now that $G=(G(t))_{t \in[0,1]}$ is such that, for some $0<\beta<2$,

$$
\mathbf{E}\left(|G(s)-G(t)|^{2}\right) \leq|s-t|^{\beta}
$$

for every $s, t \in[0,1]$. (Such a Gaussian process always admits a version with almost all sample paths continuous and Hölder of parameter $\gamma<\beta / 2$; cf., e.g., $[1,12]$; this actually also follows from the arguments developed hereafter.) By definition of $G_{n k}$, it is immediate that, for every $k, n$,

$$
\left(\mathbf{E}\left(G_{n k}^{2}\right)\right)^{1 / 2} \leq 2 \cdot 2^{n(1-\beta) / 2} .
$$

It follows from what proceeds that

$$
\mathbf{E}\left(|F|_{B}\right) \leq \mathbf{E}(|G(0)+G(1)|)+C
$$

for some constant $C$ only depending on $\beta$, possibly changing from line to line below. Similarly, one gets that, for every $m \geq 1$,

$$
\mathbf{E}\left(|H|_{B}\right) \leq C \sqrt{m} 2^{-m \beta / 2} .
$$

On the other hand, with the obvious notation,

$$
\left|\sum_{l=1}^{N} F_{l} \otimes \widetilde{F}_{l}\right|_{B \otimes B} \leq \sum_{n \leq m, k} \sum_{n^{\prime} \leq m, k^{\prime}}\left|\varphi_{n, k}\right|_{B}\left|\varphi_{n^{\prime}, k^{\prime}}\right|_{B} \mathbf{E}\left(\left|\sum_{l=1}^{N} G_{n k}^{l} \widetilde{G}_{n^{\prime} k^{\prime}}^{l}\right|\right)
$$

so that

$$
\mathbf{E}\left(\left|\sum_{l=1}^{N} F_{l} \otimes \widetilde{F}_{l}\right|_{B \otimes B}\right) \leq C\left(\sum_{n=0}^{m} 2^{n(1-\beta / 2)}\right)^{2} \sqrt{N} \leq C 2^{m(2-\beta)} \sqrt{N}
$$

where we used that by independence and (6.7),

$$
\mathbf{E}\left(\left|\sum_{l=1}^{N} G_{n k}^{l} \widetilde{G}_{n^{\prime} k^{\prime}}^{l}\right|\right) \leq 4 \sqrt{N} 2^{n(1-\beta)} .
$$

Summarizing, for every $m \geq 1$ and $N \geq 1$,

$$
\mathbf{E}\left(\left|\sum_{l=1}^{N} G_{l} \otimes \widetilde{G}_{l}\right|_{B \otimes B}\right) \leq C\left(2^{m(2-\beta)} \sqrt{N}+\sqrt{m} 2^{-m \beta / 2} N\right) .
$$

Optimizing in $m$ shows that

$$
\mathbf{E}\left(\left|\sum_{l=1}^{N} G_{l} \otimes \widetilde{G}_{l}\right|_{B \otimes B}\right) \leq C N^{\alpha}
$$

for every $(8-3 \beta) /(8-2 \beta)<\alpha<1$, some $C>0$ and all $N \geq 1$.

The uniform norm on $B=C([0,1])$ in the above argument may easily be replaced by stronger norms such as Hölder, Besov or Sobolev norms. Indeed, the 
Schauder basis decomposition is based on the property (6.5) that, for every $a_{k}$, $k=1, \ldots, 2^{n}$, and every $n \geq 0$,

$$
\left|\sum_{k=1}^{2^{n}} a_{k} \varphi_{n k}\right|_{B} \leq K 2^{-n((1 / 2)-\gamma)} \max _{1 \leq k \leq 2^{n}}\left|a_{k}\right|
$$

that thus holds with $\gamma=0$ for the uniform norm. Property (6.8) was introduced in $[27,28]$ in the investigation of small ball probabilities. As discussed there, Hölder norm of parameter $\gamma<\frac{1}{2}$ satisfies (6.8), as well as various Besov or Sobolev norms. $L^{p}$-norms, $1 \leq p<\infty$, satisfy (6.8) with $\gamma=0$. For a norm satisfying (6.8) for some $0 \leq \gamma<\frac{1}{2}$, the conclusion applies similarly provided that $\gamma<\beta / 2$. For example, for Brownian motion $\beta=1$ and any tensor norm of the Hölder norm of parameter $\gamma<\frac{1}{2}$ is exact for Wiener measure.

We thus proved the following result. We only state it for uniform and Hölder norms, but as just mentioned, it holds similarly for various Besov and Sobolev norms satisfying (6.8) with the same parameter $\gamma$. We furthermore ignore the separability questions that arise with the use of Hölder norms that are handled easily here.

THEOREM 5. Let $G=(G(t))_{t \in[0,1]}$ be a centered Gaussian process on $[0,1]$ such that, for some $0<\beta<2$, and all $s, t \in[0,1]$,

$$
\mathbf{E}\left(|G(s)-G(t)|^{2}\right) \leq|s-t|^{\beta} .
$$

(The process $G$ admits a version with almost all sample paths continuous and Hölder of parameter $\gamma<\beta / 2$.) Then any tensor norm of the uniform norm or the Hölder norm of parameter $\gamma<\beta / 2$ is exact with respect to (the distribution of) $G$.

This result includes classical Brownian motion on $C([0,1])$, but much more general Gaussian processes may be considered such as fractional Brownian motions etc. (see [1]). For example, fractional Brownian motion on $\mathbf{R}$ is a continuous Gaussian process $(G(t))_{t \in \mathbf{R}}$ such that

$$
\mathbf{E}\left(|G(s)-G(t)|^{2}\right)=|s-t|^{2 h}, \quad t, s \in \mathbf{R},
$$

where $0<h<1$ so that the preceding applies on each finite interval of $\mathbf{R}$.

Processes with values in $\mathbf{R}^{d}$ may be treated similarly. One may also investigate processes on $[0,1]^{p}$ such as Brownian sheets (see [13]). To investigate further, more general examples, we follow a procedure on a different approximation scheme based on covering numbers. To present these results, let us consider for simplicity $B=L^{\infty}(T)$ on some (finite) set $T$. Let $G=\left(G(t)_{t \in T}\right.$ be a (centered) Gaussian process on $T$ and set $d(s, t)=\left(\mathbf{E}\left(|G(s)-G(t)|^{2}\right)\right)^{1 / 2}$, $s, t \in T$. As in Section 2.2, denote, for each $\varepsilon>0$, by $N(T, d ; \varepsilon)$ the smallest number of open balls with radius $\varepsilon$ that is necessary to cover $T$. 
THEOREM 6. Let $G=(G(t))_{t \in T}$ be a (centered) Gaussian process on $T$ with $L^{2}$-pseudometric d. Assume that for constants $C>0, \beta>0$ and every $\varepsilon>0$,

$$
N(T, d ; \varepsilon) \leq C \varepsilon^{-\beta} \text {. }
$$

[The process $G$ admits a version with almost all sample paths in $L^{\infty}(T)$.] Then, any tensor norm is exact with respect to (the distribution of) $G$ on $L^{\infty}(T)$.

PROOF. The fact that $G$ admits a version with almost all sample paths in $L^{\infty}(T)$ follows from Dudley's bound (3.3). Assume for simplicity [cf. (3.1)] that $\mathbf{E}\left(\sup _{t \in T}|G(t)|^{2}\right)=1$. We may also assume without loss of generality that $T$ is finite and thus bound above any tensor norm (the projective one, for example) on $L^{\infty}(T) \otimes L^{\infty}(T)$. We use a chaining approximation of $G$. For any integer $k \in \mathbf{Z}$, denote by $\left\{A_{i}\right\}$ a finite partition of $T$ in $N\left(2^{-k}\right)$ pieces where $N(\varepsilon)=N(T, d ; \varepsilon)$ is the $\varepsilon$-covering of $T$ with respect to $d$. Denote by $s_{i}$ a fixed point in $A_{i}$ and consider the process $F=(F(t))_{t \in T}$ defined by

$$
F(t)=G\left(s_{i}\right) \quad \text { if } t \in A_{i} .
$$

The usual chaining argument in the proof of the entropic bound (3.3) on the supremum of Gaussian processes shows that (cf. [19], page 306)

$$
\mathbf{E}\left(\sup _{t \in T}|G(t)-F(t)|\right) \leq C \sum_{m>k} 2^{-m} \sqrt{\log N\left(2^{-m}\right)}=\varepsilon(k)
$$

where $C>0$ is a numerical constant possibly changing from line to line below. Due to the hypothesis, $\varepsilon(k) \rightarrow 0$ as $k \rightarrow \infty$ so that we let $\varepsilon(k) \leq 1$ below. Set $H(t)=G(t)-F(t), t \in T$, and follow the decomposition (6.3) used in the proof of (6.2) and Theorem 5. Again, if $G_{l}, \widetilde{G}_{l}$, resp. $F_{l}, \widetilde{F}_{l}, H_{l}, \tilde{H}_{l}, l \geq 1$, are independent copies of $G$, resp. $F, H$, we write similarly

$$
\sum_{l=1}^{N} G_{l} \otimes \widetilde{G}_{l}=\sum_{l=1}^{N} F_{l} \otimes \widetilde{F}_{l}+\sum_{l=1}^{N} F_{l} \otimes \tilde{H}_{l}+\sum_{l=1}^{N} H_{l} \otimes \widetilde{F}_{l}+\sum_{i=1}^{N} H_{l} \otimes \tilde{H}_{l} .
$$

By the triangle inequality and (6.9),

$$
\mathbf{E}\left(\left|\sum_{l=1}^{N} F_{l} \otimes \tilde{H}_{l}\right|_{L^{\infty}(T) \otimes L^{\infty}(T)}\right) \leq C \varepsilon(k) N .
$$

Similar bounds hold for $\sum_{l=1}^{N} H_{l} \otimes \widetilde{F}_{l}$ and $\sum_{i=1}^{N} H_{l} \otimes \tilde{H}_{l}$. On the other hand,

$$
\left|\sum_{l=1}^{N} F_{l} \otimes \widetilde{F}_{l}\right|_{L^{\infty}(T) \otimes L^{\infty}(T)}=\sup \sum_{i, j=1}^{N\left(2^{-k}\right)}\left(\sum_{l=1}^{N} G_{l}\left(s_{i}\right) \widetilde{G}_{l}\left(s_{j}\right)\right)\left\langle I_{A_{i}}, \varphi\left(I_{A_{j}}\right)\right\rangle
$$

so that by the scalar case

$$
\mathbf{E}\left(\left|\sum_{l=1}^{N} F_{l} \otimes \widetilde{F}_{l}\right|_{B \otimes B}\right) \leq N\left(2^{-k}\right)^{2} \sqrt{N}
$$


[recall that $\left.\mathbf{E}\left(\sup _{t \in T}|G(t)|^{2}\right)=1\right]$. It thus follows that

$$
\mathbf{E}\left(\left|\sum_{l=1}^{N} G_{l} \otimes \widetilde{G}_{l}\right|_{L^{\infty}(T) \otimes L^{\infty}(T)}\right) \leq N\left(2^{-k}\right) \sqrt{N}+C \varepsilon(k) N .
$$

Since $N\left(2^{-k}\right) \leq C 2^{\beta k}$, it is easily seen that $\varepsilon(k) \leq C 2^{-k} \sqrt{\log 2^{k}}$ from which we conclude that

$$
\mathbf{E}\left(\left|\sum_{l=1}^{N} G_{l} \otimes \widetilde{G}_{l}\right|_{L^{\infty}(T) \otimes L^{\infty}(T)}\right) \leq C N^{\alpha}
$$

for every $(1+4 \beta) /(2+4 \beta)<\alpha<1$. The proof of Theorem 6 is complete.

\section{REFERENCES}

[1] AdLer, R. J. (1990). An Introduction to Continuity, Extrema and Related Topics for General Gaussian Processes. IMS, Hayward, CA.

[2] Bass, R. F., Hambly, B. M. and Lyons, T. J. (1999). Extending the Wong-Zakai theorem to reversible Markov processes. Preprint.

[3] BRZEŹNIAK, Z. (1997). On stochastic convolution in Banach spaces and applications. Stochastics Stochastics Rep. 61 245-295.

[4] BrZeŹniaK, Z. and CARroll, A. (2000). Approximations of the Wong-Zakai type for stochastic differential equations in $M$-type 2 Banach spaces with applications to loop spaces. Preprint.

[5] Brzeźniak, Z. and Elworthy, D. (1999). Stochastic differential equations on Banach manifolds. Preprint.

[6] Capitaine, M. and Donati-Martin, C. (2001). The Lévy area for the free Brownian motion. J. Funct. Anal. 179 153-169.

[7] Chevet, S. (1977). Un résultat sur les mesures gaussiennes. C. R. Acad. Sci. Paris Sér. I Math. 284 441-444.

[8] Coutin, L. and QIAN, Z. (2002). Stochastic analysis, rough path analysis and fractional Brownian motion. Probab. Theory Related Fields 122 108-140.

[9] DA Prato, G. and ZABCZYK, J. (1992). Stochastic equations in infinite dimensions. In Encyclopedia of Mathematics and Its Applications 44. Cambridge Univ. Press.

[10] Dudley, R. M. (1967). The sizes of compact subsets of Hilbert space and continuity of Gaussian processes. J. Funct. Anal. 1 290-330.

[11] Fernique, X. (1970). Intégrabilité des vecteurs gaussiens. C. R. Acad. Sci. Paris Sér. I Math. 270 1698-1699.

[12] Fernique, X. (1975). Regularité des trajectoires des fontions aléatoires gaussiennes. Ecole d'ete probabilites de St. Flour IV. Lecture Notes in Math. 480 1-96. Springer, New York.

[13] Goodman, V. and Kuelbs, J. (1991). Rates of clustering for some Gaussian self-similar processes. Probab. Theory Related Fields 88 47-75.

[14] Gross, L. (1965). Abstract Wiener spaces. Proc. Fifth Berkeley Symp. Math. Statist. Probab. 2 31-42. Univ. California Press, Berkeley.

[15] Gross, L. (1970). Lecture on Modern Analysis and Applications II. Lecture Notes in Math. 140. Springer, New York.

[16] Hambly, B. M. and Lyons, T. J. (1998). Stochastic area for Brownian motion on Sierpinski gasket. Ann. Probab. 26 132-148. 
[17] Kuo, H.-H. (1975). Gaussian Measures in Banach Spaces. Lecture Notes in Math. 436. Springer, New York.

[18] Landau, H. J. and Shepp, L. A. (1970). On the supremum of a Gaussian process. Sankhya Ser. A 32 369-378.

[19] Ledoux, M. and Talagrand, M. (1991). Probability in Banach Spaces: Isoperimetry and Processes. Springer, New York.

[20] Ledoux, M. (1996). Isoperimetry and Gaussian Analysis. Ecole d'Eté de Probabilités de St. Flour. Lecture Notes in Math. 1648 165-294. Springer, New York.

[21] LI, W. and LINDE, W. (1999). Approximation, metric entropy and small ball estimates for Gaussian measures. Ann. Probab. 27 1556-1578.

[22] Lifshits, M. A. (1995). Gaussian Random Functions. Kluwer, Dordrecht.

[23] LyONS, T. J. (1998). Differential equations driven by rough signals. Rev. Mat. Iberoamericana 14 215-310.

[24] LYONS, T. J. and QIAN, Z. (1998). Flows of diffeomorphisms induced by a geometric multiplicative functionals. Probab. Theory Related Fields 112 91-119.

[25] Lyons, T. J. and QIAN, Z. (2000). System Control and Rough Paths. Oxford Univ. Press.

[26] Sippilainen, E.-M. (1993). A pathwise view of solutions of stochastic differential equations. $\mathrm{Ph} . \mathrm{D}$. dissertation, Univ. Edinburgh.

[27] Stolz, W. (1993). Une méthode élémentaire pour l'évaluation de petites boules browniennes. C. R. Acad. Sci. Paris Sér. I Math. 316 1217-1220.

[28] Stolz, W. (1996). Some small ball probabilities for Gaussian processes under non-uniform norms. J. Theoret. Probab. 9 613-630.

[29] Walsh, J. B. (1986). An introduction to stochastic partial differential equations. École d'été de probabilités de St. Flour XIV. Lecture Notes in Math. 1180 265-437. Springer, New York.

M. LEDOUX

Z. QIAN

LABoratoire de Statistique et Probabilities

DÉPARTEMENT DE MATHÉMATIQUES

C.N.R.S. ET UNIVERSITÉ PAUL-SABATIER

118 , ROUTE DE NARBONNE

31062 TOULOUSE

FRANCE

E-MAIL: ledoux@math.ups-tlse.fr

qian@math.ups-tlse.fr
T. J. LYONS

MATHEMATICAL InSTITUTE

UNIVERSITY OF OXFORD

24-29 ST-GILES, OXFORD OX1 3LB

UNITED KINGDOM

E-MAIL: tlyons@maths.ox.ac.uk 